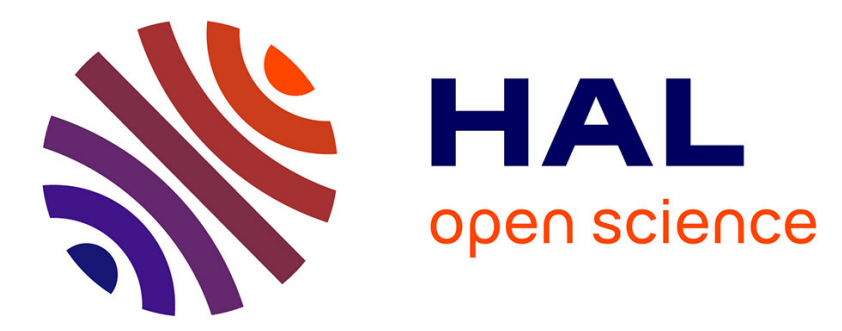

\title{
Les Yasodharàsrama, marqueurs d'empire et bornes sacrées : conformité et spécificité des stèles digraphiques khmères de la région de Vat Phu
}

\author{
Julia Estève, Dominique Soutif
}

\section{- To cite this version:}

Julia Estève, Dominique Soutif. Les Yasodharàsrama, marqueurs d'empire et bornes sacrées: conformité et spécificité des stèles digraphiques khmères de la région de Vat Phu. Bulletin de l'Ecole française d'Extrême-Orient, 2010, 97 (1), pp.331 - 355. 10.3406/befeo.2010.6137 . halshs-02550819

\section{HAL Id: halshs-02550819 \\ https://shs.hal.science/halshs-02550819}

Submitted on 22 Apr 2020

HAL is a multi-disciplinary open access archive for the deposit and dissemination of scientific research documents, whether they are published or not. The documents may come from teaching and research institutions in France or abroad, or from public or private research centers.
L'archive ouverte pluridisciplinaire HAL, est destinée au dépôt et à la diffusion de documents scientifiques de niveau recherche, publiés ou non, émanant des établissements d'enseignement et de recherche français ou étrangers, des laboratoires publics ou privés. 


\section{Les Yasodharàsrama, marqueurs d'empire et bornes sacrées :} conformité et spécificité des stèles digraphiques khmères de la région de Vat Phu

Julia Estève, Dominique Soutif

\section{Citer ce document / Cite this document :}

Estève Julia, Soutif Dominique. Les Yasodharàsrama, marqueurs d'empire et bornes sacrées : conformité et spécificité des stèles digraphiques khmères de la région de Vat Phu. In: Bulletin de l'Ecole française d'Extrême-Orient. Tome 97-98, 2010. pp. 331-355;

doi : https://doi.org/10.3406/befeo.2010.6137

https://www.persee.fr/doc/befeo_0336-1519_2010_num_97_1_6137

Fichier pdf généré le 08/11/2019 


\title{
Résumé
}

L'épigraphie de Yasovarman ler commémore la création, par ce roi, d'une centaine d"àsrama dans l'ensemble de son empire. Pourtant, malgré leur importance manifeste, nous serions bien en peine d'identifier ces lieux sans doute essentiellement construits en matériaux légers, s'ils n'étaient signalés par des inscriptions qui célèbrent leur fondation

- les stèles digraphiques -, dont seize ont été identifiées à ce jour. Nous nous intéresserons ici à deux d'entre-elles qui proviennent de la région de Vat Phu : une stèle complète découverte dans le sanctuaire de Houay Tomo (K. 362) et deux groupes de fragments découverts, à proximité de Vat Phu, à trente ans d'intervalle, dont on démontrera qu'ils appartiennent à une même stèle ( $\mathrm{K}$. 1005). Une fois reconstitué, le texte permet d'associer l'àsrama au dieu de Vat Phu, Bhadresvara. L'étude de ces textes, et notamment de leur spécificité par rapport aux autres stèles digraphiques, nous permettra de souligner l'importance de ces institutions religieuses, en particulier leur rôle de vecteur de transmission du pouvoir royal à un moment clé de la constitution de l'empire khmer. Enfin, nous verrons que ces àsrama étaient associés à des sanctuaires vénérables, dont bien souvent, la fondation remonte au moins à la période préangkorienne. Partant de ce constat et des différents rôles assignés aux àsrama, nous nous attacherons à montrer en quoi la connaissance de ces fondations permettra, à terme, de dresser une carte tant politique que religieuse de l'empire khmer à la fin du IXe siècle.

\begin{abstract}
Yasovarman l's epigraphy commemorates the establishment by this king of some 100 âsramas throughout his empire. However, despite their key functions, these sites remain difficult to identify as they were mainly built with non-durable materials. It is only thanks to inscriptions (digraphic steles) praising their foundations that their existence was brought to light. As of today, 16 such digraphic steles have been identified. This article focuses on two steles found in the Vat Phu area: one entire stele unearthed at the Houay Tomo shrine (K. 362) and two clusters of fragments found near Vat Phu over a 30-year period and proved to belong to the same àsrama stele (K. 1 005). After it was pieced back together, the latter showed the association of this àsrama with Bhadresvara, the Vat Phu god. The study of these texts and of their contents in relation with other digraphic steles allows us to highlight the major role played by these religious establishments, particularly when acting as vectors of the central power at a key moment in the establishment of the Khmer empire. Finally, it will display the association of these âsramas with particularly venerable sanctuaries, the foundations of which are often dated to pre-Angkorian times. Based on these findings and on the different roles attributed to âsramas, this article shows how an in-depth knowledge of these foundations will eventually enable us to draw up a political and religious map of the late 9th century Khmer Empire.
\end{abstract}




\title{
Les Yaśodharāśrama, marqueurs d'empire et bornes sacrées
}

\author{
Conformité et spécificité des stèles digraphiques khmères \\ de la région de Vat Phu
}

\author{
Julia Estève \& Dominique SoutiF ${ }^{1}$
}

\begin{abstract}
Si l'on en croit les quatre inscriptions du Baray oriental et l'inscription de Lolei ${ }^{2}$, cent $a \overline{a s} r a m a$ émaillaient le territoire khmer à la fin du $\mathrm{IX}^{\mathrm{e}}$ siècle de notre ère. Ces établissements ${ }^{3}$ servaient à la fois de lieux de transmission du savoir, de centres de retraite spirituelle et de gîtes d'étape : ils accueillaient les pèlerins de toutes provenances et obédiences, recevaient le roi ou sa cour en déplacement et abritaient une communauté religieuse dotée de règles spécifiques, placée sous la responsabilité d'un supérieur ${ }^{4}$.

Les áśrama ponctuaient le paysage de l'empire angkorien encore en constitution, depuis le sud du Laos jusqu'au delta du Mékong (fig. 1). Leur construction est censée avoir commencé dès la première année d'accession au trône de leur fondateur, le roi Yaśovarman I ${ }^{\mathrm{er}}$ (r. 889-ca. - 910 de notre ère). Allant de pair avec chacune de ces structures, une inscription de fondation était érigée à proximité, pour en rappeler l'affiliation religieuse et les règles de fonctionnement. Le corpus épigraphique du roi forme ainsi un ensemble important au sein duquel deux groupes nous intéressent plus particulièrement : les inscriptions de fondation des quatre äśrama d'Angkor ${ }^{5}$ et les inscriptions digraphiques qui commémoraient la fondation des āśrama de province ${ }^{6}$.
\end{abstract}

1. Cette étude entre dans le cadre du programme de recherche Yaśodharāśrama (EFEO/EPHE/ APSARA). Nous tenons à remercier ici David Bazin, Gerdi Gerschheimer, Christine Hawixbrock, Michel Lorrillard, Bertrand Porte et Christophe Pottier pour l'aide qu'ils nous ont apportée.

2. K. 280-283, st. XXXIII (ISCC, p. 434, 442); K. 323, st. XLVI (ISCC, p. 398, 406).

3. La traduction généralement admise pour le terme āśrama, « ermitage », ne rend pas vraiment justice à cette institution, car elle tend à réduire son importance et la diversité de ses attributions. Cependant, nous l'utiliserons par commodité.

4. Les responsables des āśrama d'Angkor sont désignés par le composé kulādhyakșa (K. 279, st. LII ; Cœdès 1932, p. 90) et ceux des āśrama de province par kulapati (K. 95, st. XLV; ISCC, p. 368).

5. L'inscription K. 290 de Tep Pranam, qui proviendrait du Prasat Ong Mong (Cœdès 1908, p. 203 ; IC III, p. 231 (cf. n. 31) ; l'inscription K. 701 (Cœdès 1932, p. 88) du Prasat Komnap sud ; l'inscription K. 279 (ISCC, p. 418 ; Codès 1932, p. 85) de Prei Prasat, et l'inscription K. 1228, découverte sur le site de Kôk Ta Sœng, mais qui proviendrait du Prasat Komnap nord (Estève 2009, p. 339-344 ; Pottier 2003 ).

6. Les inscriptions digraphiques comportent quasiment le même texte sur leurs deux faces mais dans deux écritures différentes. Elles constituent à ce jour un groupe de seize stèles dont la liste est donnée dans un tableau (fig. 2). L'édition de référence pour ces inscriptions repose sur deux d'entre elles, K. 95 et K. 362 (ISCC, p. 363-376, 390 ; Cœdès 1932, p. 108). Il faut toutefois ajouter à cette liste l'inscription K. 323 de Lolei, également digraphique, mais dont le texte, plus long, commémore l'installation des quatre divinités de ce temple, et non la fondation d'un àsrama. 
Chacun des àśrama d'Angkor présente la particularité d'être dédié de façon exclusive à un courant religieux : vishnouisme, bouddhisme et deux mouvements probablement shivaïtes ${ }^{7}$. Les supérieurs des āśrama se partageaient la protection du Yaśodharatațāka, à savoir le Baray oriental, immense réservoir dont le rôle était central pour la vie économique et religieuse de la capitale.

Les āśrama de province accueillaient quant à eux toutes les communautés religieuses en leurs murs, sans discrimination, mais ils étaient dédiés à une seule divinité ${ }^{8}$. Signalés par les « bornes sacrées » que sont les inscriptions digraphiques, ces ermitages étaient probablement construits pour l'essentiel en matériaux légers et sont désormais difficilement identifiables. Onze de ces inscriptions de bornes sacrées ont été publiées par Auguste Barth et Abel Bergaigne dès 1893, et leur nombre s'est enrichi au fil des ans. L'inscription K. 479 fut d'abord signalée par Étienne Lunet de Lajonquière en Thaïlande (1911, p. 456-457). L'inscription K. 1005 fut ensuite découverte au Laos à une date inconnue - nous y reviendrons. En 1978, ce sont les inscriptions K. 1092 et K. 1093 qui furent retrouvées en Thaïlande (Claude Jacques, comm. pers.), puis en 1994 l'inscription K. 1223, au Cambodge. Enfin, plusieurs fragments attendent encore d'être inventoriés ; ils proviennent également du Laos. Ces textes viennent étoffer notre savoir concernant la liste des divinités d'élection de ces établissements religieux de province et permettent ainsi d'esquisser une carte religieuse de la fin du Ix $x^{e}$ siècle.

Jayavarman II (r. 802 - ca. 835), dont le règne débuta dans le sud-est du Cambodge moderne, est le premier roi des Khmers appelé de façon assurée cakravartin ou « monarque universel ". Il est célèbre dans l'historiographie pour avoir, dans une certaine mesure, pacifié et unifié le pays, et pour avoir établi sa capitale à quinze kilomètres au sud-est du futur site d'Angkor, à Hariharālaya. Pourtant, bien que son règne marque traditionnellement le début de la période angkorienne, il semble que ce soit Yaśovarman $I^{\text {er }}$ qui installa le premier sa capitale à Angkor même. En effet, les successeurs de Jayavarman II, Jayavarman III et Indravarman ${ }^{\mathrm{I} r}$, demeurèrent à Hariharālaya. L'extension du royaume que suggèrent le nombre présumé et la répartition géographique des āśrama, tend à montrer en outre que Yaśovarman $I^{\text {er }}$ contribua grandement à la constitution de l'empire khmer, tout en portant la centralisation du pouvoir à un niveau supérieur. Les áśrama ont vraisemblablement joué le rôle de vecteurs privilégiés pour cette entreprise : étant donné leur vocation multiforme de lieu de culte et d'enseignement, ils constituaient le moyen idéal pour étendre la «culture khmère » et l'imposer. Les stèles de fondation qui les accompagnaient, en attribuant la construction de l'ermitage au roi, concouraient à accroître sa renommée et à rappeler sa puissance.

Même si l'on est en droit de se demander si le nombre de cent àśrama n'est pas un peu exagéré ou symbolique, il n'en demeure pas moins que ceux-ci jouaient à la fois le rôle de marqueurs, matérialisant l'extension de l'empire à la fin du IX $x^{c}$ siècle, et des

7. L'inscription K. 290 rappelle la fondation d'un äśrama bouddhique, le saugatāsrama : l'āsirama vishnouite, ou vaișnavāiśrama, est répertorié dans l'inscription K. 701 . Les deux äśrama shivaïtes sont le brāhmanāśrama dont la fondation est commémorée dans l'inscription K. 279, et le māheśvaräśrama, dans l'inscription K. 1228. On notera que les inscriptions de fondation des äśrama d'Angkor n'utilisent pas le composé générique vaśodharāśrama mais des composés rappelant leur obédience respective.

8. On notera que deux de ces inscriptions digraphiques sont dédiées au Gaṇeśa de Candanādri, sans qu'il soit possible de déterminer s'il s’agit d'une crreur ou si ce toponyme et la divinité qui lui est associée étaient attestés dans deux endroits différents (sur ce sujet, cf. ISCC, p. 362, n. 2). 
bornes sacrées. Ils imprimaient du sceau de Yaśovarman Irr les terres situées aux marges, un sceau apposant également les traits culturels et religieux khmers.

Cette étude porte plus particulièrement sur le nord-est de l'empire : elle se consacre aux stèles digraphiques provenant du Laos (fig. 3) dans le but de mettre en lumière leur conformité avec les autres stèles digraphiques, mais ćgalcment lcurs spécificitćs.

Afin de prendre la mesure de ces documents, il convient d'abord de rappeler le contenu commun des inscriptions digraphiques. Outre les éléments qui les singularisent, leur intérêt réside dans le fait qu'elles répètent quasiment à l'identique, dans toutes les régions de l'empire, un texte dont la composition générale est fixe. Il serait inutile de reproduire ici de façon détaillée les contenus des inscriptions digraphiques du Laos, la majeure partie en ayant déjà été éditée et traduite par A. Barth et $\mathrm{A}$. Bergaigne (ISCC, p. 346-390) ${ }^{9}$, puis corrigée par George Codès (1932, p. 108-112); nous n'en citerons donc que quelques passages inédits ou pertinents.

\section{Le commanditaire : Yaśovarman Ier $^{\text {r }}$}

Après une stance d'invocation à la triade brahmanique (Śiva, Viṣnu et Brahmā), les quinze suivantes ${ }^{10}$ sont consacrées à la généalogie de Yaśovarman $\mathrm{I}^{\mathrm{er}}$, la plus détaillée que l'épigraphie livre sur ce roi. Elle s'étend sur six générations, couvrant d'abord sa lignée maternelle, puis sa lignée paternelle, le pouvoir étant transmis au Cambodge par succession matrilinéaire. L'auteur de l'inscription s'applique à confirmer la légitimité du roi en soulignant la noblesse de ses origines, le rattachant d'abord aux lignées de seigneurs ou souverains de Śambhupura, Vyādhapura et Aninditapura, puis rappelant, par le jeu des alliances, ses liens de parenté avec Jayavarman II $^{11}$.

Suivent dix-neuf stances de panégyrique du roi, où sont célébrées ses vertus de combattant, sa sagesse, sa beauté, sa gloire, ainsi que la générosité de ses dons et de ses sacrifices aux dieux. Deux de ses plus célèbres fondations sont également mentionnées : le temple de Lolei, consacré à ses parents et à ses grands-parents maternels, et le Yaśodharatațāka, c'est-à-dire le Baray oriental, dont on a déjà évoqué l'importance et le lien avec les quatre áśrama de la capitale.

9. L’inscription de référence utilisée par A. Bergaigne et A. Barth pour établir l'édition de ces inscriptions est la K. 95, qui présente un excellent état de conservation. Elle provient du site de Phnom Preah Bat (province de Kompong Cham). Pour ce qui concerne les autres stèles, en dehors de la stance XXXVI, différente pour chaque inscription, ces auteurs n'évoquent, dans leur publication, que les rares variantes ou fautes orthographiques.

10. Toutes ces inscriptions présentent une « mise en pierre » différente (entre elles et sur chaque face) qui interdit de renvoyer aux lignes où sont notées les stances en question. De façon générale, elles comprennent cinquante stances en sanskrit pour la face $A$ et quarante-neuf stances en sanskrit, plus une phrase en khmer, pour la face B, mais on constate certaines variantes; nous reviendrons sur cette composition.

11. Yaśovarman ler était le descendant, par sa mère, de "l'oncle maternel de l'oncle maternel de la mère » de Jayavarman II. Du côté de son père, il était, semble-t-il, l'arrière petit-fils de l'oncle maternel de la femme de Jayavarman II (ISCC, p. 357-359). Pour ténue que soit cette parenté, le lien est donc établi avec la précédente famille royale souveraine du pays khmer et il n'est pas surprenant que cet argument ait joué en faveur de la légitimité du roi, quand on sait à quel point l'épigraphie angkorienne souligne l'importance du règne de Jayavarman II. C'est par exemple à ce règne que l'on fait remonter la propriété d'une terre ou d'une charge quand on souhaite affirmer son authenticité (cf. par exemple l'inscription K. 956, I. 7-24; IC VII, p. 130-133). 


\section{Dédicace}

La stance XXXVI constitue le pivot du texte des stèles digraphiques dans la mesure où elle précise enfin leur objet : la fondation, par le roi, d'un àśrama. Elle marque donc le début du śāsana, l'«édit » proprement dit. Les deux premiers pāda du vers sont communs à toutes les inscriptions :

yaśodharāśrame datte śrimmatīndvekamīrttibhị

La première traduction de ce passage, proposée par A. Bergaigne, restituait d'office une affiliation shivaïte pour ce qui était appelé yaśodharāśrama (ou " ermitage de Yaśovarman »), et dissociait, de ce fait, la fondation du reste de la stance :

«Après avoir donné (à Śiva) le vénérable Yaśodharāśrama en lune, un, formes (de Śiva), ...».

En cela, elle avait créé une certaine confusion en laissant supposer qu'un unique et " splendide " "Yaśodharāśrama avait été fondé, sans doute à Angkor ${ }^{13}$. Dans un article faisant écho aux travaux archéologiques conduits par Georges Trouvé sur les àśrama d'Angkor, G. Cœdès corrigea cette interprétation. En premier lieu, il observa que dans ces inscriptions rien ne permet de rattacher ce supposé Yaśodharāśrama à un culte shivaïte. De plus, il releva que l'on y exprime avec insistance la possibilité pour ces institutions d'accueillir des religieux de diverses obédiences, tant des brähmana que des vaisnava et des śaiva (st. XLIII). En second lieu, la découverte de trois inscriptions de fondation d'áśrama par Yaśovarman I ${ }^{\text {er }}$ à Angkor permit à G. Coedès de démontrer qu'il ne fallait pas chercher un unique Yaśodharāśrama dans la capitale. Le composé yaśodharāśrama de la première partie de la stance renvoie en définitive à " une série d'ermitages identiques ", fondés simultanément dans plusieurs régions de l'empire : les ermitages de nos inscriptions digraphiques, dont le nombre s'élèverait à cent. Le terme est à comprendre comme désignant à chaque fois l'institution dont la stèle entérine la fondation : chacun de ces āśrama est appelé Yaśodharāśrama. La traduction des deux premiers pāda de la stance XXXVI que propose G. Cœdès est alors la suivante :

« Le vénérable Yaśodharāśrama ayant été donné (ici) en lune (un), un, formes (huit)

[soit 811 de l'ère śaka]... ${ }^{14}$

Comme on le voit, l'édit commémorant la décision royale de fonder ces ermitages de façon plus vraisemblable que leur fondation simultanée proprement dite - est donc

12. Cette qualification de l'ermitage comme « splendide » était une surinterprétation du terme śrmat (signifiant « vénérable »), due à Étienne Aymonier (1904, p. 47).

13. Si l'on prend l'exemple de l'inscription de référence K. 95 , le passage dans son entier donnait, ainsi, d'après la traduction de Bergaigne revue par Barth :

laśsodharāsrame datte śrimmatīndvekamūirttibhih candanādriganeśäya śāsanam sa vyadhād idam \|

"Après avoir donné (à Śiva) le vénérable Yaśodharāśrama en lune, un, formes (de Śiva) (811 śaka), il a rendu ce décret pour lc Gaṇcśa de Candanādri (Mont du Santal). "

14. En reprenant notre exemple (l'inscription K. 95), la stance se traduit donc comme suit : « Le vénérable Yaśodharāśrama ayant été donné (ici) en lune (un), un, formes (huit), il a fait cet édit pour le Gaṇeśa de Candanādri (Mont du Santal) ». 
daté de 811 saka ${ }^{15}$, soit 889 ou 890 de notre ère, c'est-à-dire au moment de l'avènement de Yaśovarman $\mathrm{I}^{\mathrm{er}}$, ou en tout cas peu de temps après.

La date de la fondation est déjà intéressante en soi mais les deux pāda suivants complètent avantageusement cette première partie du śloka. Il s'agit là de la variante majeure entre les textes, puisque ces pāda livrent le nom de la divinité en faveur de laquelle l'áśrama a été fait et auquel il était rattaché, indiquant de ce fait son affiliation religieuse. Sur les seize stèles de fondation d'āśrama de province, onze stances de dédicace ont été conservées ${ }^{16}$. Elles mettent en évidence la variété des divinités présentes sur le sol khmer. Si la majorité des occurrences qui nous sont parvenues font référence à des sanctuaires shivaïtes, l'inscription K. 47 de Tuol Vat Kdei Kandal (province de Prei Veng), dédiée à Nārāyaṇa, témoigne de l'existence d'un ermitage vishnouite au sud-est de l'empire.

Considérons à présent les dédicaces des inscriptions digraphiques connues au Laos.

\section{L'āśrama de Houay Tomo (l'inscription K. 362)}

Au Laos, ce sont deux dédicaces de ce type qui sont conservées, toutes deux shivaïtes. Celle de la stèle digraphique de Houay Tomo (K. 362 ; fig. 8) a été traduite par A. Bergaigne (ISCC, p. 389) ; elle est dédiée à Rudrāṇī, la parèdre de Rudra. Il est difficile d'affirmer avec certitude que la stèle provient bien de ce site, puisqu'elle n'était déjà plus en place lors de sa découverte. É. Aymonier a toutefois précisé qu'à proximité, une fosse creusée par des pilleurs pouvait signaler son emplacement d'origine (1901, p. 171).

Convaincu du fait que la stèle provenait bien de Houay Tomo, Oscar Nalesini, qui a mené récemment une étude du site, a essayé de trouver des indices de la confession du sanctuaire dans le corpus épigraphique, afin d'établir un lien probant entre Houay Tomo et Rudrāṇī. Il a, par exemple, remarqué que l'inscription K. 300 d'Angkor Vat évoque à la fois une offrande au Śiva Bhadreśvara et à la Gañga de la ville de Lingapurī (= Lingapura ${ }^{17}$, la ville du site de Vat Phu, dont Bhadreśvara est la divinité principale ; cf. infra) et mentionne l'apparition d'une triade Śiva-Bhavānī-Jāhnavī (st. LXII, LXV et XXV, XXVI ; ISCC, p. 570, 574, 581, 585). Il établit alors un système d'équivalences :

«It is worth noting the correspondence of this triad with the gods who might have been worshipped at Huei Thamô: besides Śiva, Bhavānī coincides with Rudrānī, being a manifestation of Umā bearing one of the eight names of Rudra, while Jāhnavī (or Gangāa) is the ocean surrounding the mountain Meru, just as the Mekong and its tributary river "surrounded" the sanctuary of Huei Thamô. » (Nalesini 2000, p. 130)

15. On notera que ces stèles, portant la date de 811 śaka, évoquent à la stance XXXII la fondation du temple de Lolei qui semble dater, quant à elle, de 815 śak, moment même où sont effectuées les grandes donations royales qui devaient assurer son fonctionnement (NIC II-III, p. 65-88; Soutif 2009, p. 449-543). Il faut donc en conclure que la rédaction des inscriptions digraphiques est postérieure d'au moins quatre ans à l'entreprise de fondation des äśrama qu'elles commémorent.

16. Les dédicaces conservées sont rassemblées dans le document $1:$ les deux premiers pāda présentent deux variantes : śāsanañ krtavān iti (dans K. 42, K. 223, K. 309, K. 362 et K. 1005) et śāsanam sa vyadhād idam (dans K. 47, K. 57, K. 95, K. 101, K. 110 et K. 346). Selon G. Cœdès, les variantes observées sont « conditionnées par la scansion du nom de la divinité » (1932, p. 109). D’après G. Gerschheimer (comm. pers. ; cf. n. 23), la première variante expliquerait l'interprétation de Bergaigne et de Barth ; en revanche, la deuxième permettrait d'envisager une autre traduction. Une étude globale de l'ensemble de ce corpus est nécessaire pour statuer sur ce point.

17. Plusieurs villes portent ce nom au Cambodge, mais le fait que Bhadreśvara, la divinité de Vat Phu, soit mentionné à la stance LXV encourage cette identification. 
Il est possible que les sanctuaires de la région de Vat Phu aient rappelé une triade Śiva-Bhavānī-Jāhnavī, mais outre le fait que cette inscription du XIII ${ }^{\mathcal{B}}$ siècle est bien postérieure à la fondation de ces sites et que le lien entre les stances relevées est loin d'être si évident ${ }^{18}$, il nous semble qu'elle n'apporte aucun élément probant qui permette de déterminer si le temple de Houay Tomo accueillait ou non cette Rudrāṇī ${ }^{19}$. Bhavānī n'est en effet autre qu'Umā, mais comme Kamaleswar Bhattacharya le rappelait déjà en 1961 (dans un passage que Nalesini omet de citer), la triade Śiva-Umā-Gañgā est une représentation iconographique très répandue en Inde et également bien connue au Cambodge à cette époque ${ }^{20}$.

Un argument plus convaincant serait à prendre en considération : de fait, les àśrama de Yaśovarman $I^{\text {er }}$ furent manifestement associés à des sanctuaires particulièrement vénérables et les travaux de O. Nalesini à Houay Tomo ont certainement permis de montrer l'importance de ce site dans la province de Champassak. Ce temple est longtemps resté peu étudié et ce, en dépit du plan qu'É. Lunet de Lajonquière avait publié, plan pour le moins surprenant pour un sanctuaire khmer, et qui fut très tôt critiqué par $\mathrm{H}$. Parmentier (Lunet de Lajonquière 1907, fig. 22, p. 65 ; Parmentier 1913, p. 49). Malgré l'état de ruine avancé du site, les levés topographiques réalisés en 1999 par la mission archéologique italienne au Laos ont permis de mettre en évidence un temple étendu, présentant plusieurs phases de construction. On notera que le temple est précédé d'une chaussée bordée de bornes en grès, élément qu'on ne retrouve que dans les ensembles de grande taille (Nalesini 2000, p. 123 ; fig. 2 et 3, p. 124-125).

En l'absence de fouilles ${ }^{21}$, peu d'éléments permettent toutefois de déterminer avec certitude la date de fondation du sanctuaire ou encore son obédience. En effet, aucune autre inscription n'y a malheureusement été découverte et la statuaire comme les basreliefs ne donnent que peu d'indications.

On rappellera cependant que plusieurs linteaux ont été retrouvés sur le site dont un, au moins, peut être rattaché au style du Bakheng, et donc au règne de Yaśovarman I ${ }^{\text {er }}$ (fig. 4). Mais ce linteau ne témoigne, sans doute, que de transformations du sanctuaire réalisées sous son règne. En effet, la présence d'un canal d'évacuation des eaux lustrales orné d'une tête de makara, élément essentiellement préangkorien dont la disparition se situe, au plus tard, autour du style de Preah Kô, suggère une fondation plus ancienne (Boisselier 1966, p. 216-217 ; fig. 5) 22. Le motif décoratif central de ce linteau, Indra sur Airãvata, n'apporte aucun indice concernant l'obédience du temple. Un autre linteau, plus difficile à dater, mais probablement attribuable au $\mathrm{X}^{\mathrm{c}}-\mathrm{XI}^{\mathrm{c}}$ siècle, laisse deviner un Śiva sur le taureau et peut-être un Śiva dansant (fig. 6). Enfin, une curieuse borne à base carrée dont deux faces opposées de la partie supérieure sont ornées de visages sculptés et les deux autres de ce que l'on peut considérer comme des linga - dont un détruit - a parfois été interprétée

18. La triade dont il est question dans la stance XXVI est une apparition « ayant pris une forme sensible » et « emplissant le ciel de sa majesté ».

19. Nous renvoyons le lecteur à la note 46 p. 345 pour plus d'éléments sur cette divinité féminine.

20. Plusieurs représentations de ce type furent, par exemple, retrouvées à Bakong (cf. Bhattacharya 1961, p. 85).

21. À notre connaissance, les seuls autres travaux archéologiques réalisés récemment sur ce site sont les prospections de Marielle Santoni et Vengkéo Souksavatdy, qui ont notamment permis de collecter de grandes quantités de céramique en surface (1999[a]. p. 197).

22. On notera toutefois que, selon $O$. Nalesini, aucun bâtiment observé sur le site ne suggère une occupation préangkorienne; en attendant une étude archéologique plus poussée, on peut donc également supposer que les quelques éléments de statuaire préangkoriens ont été déplacés (2000, p. 131-132). 
comme un caturmukhalinga ou «linga à quatre visages » préangkorien (op. cit., p. 288 et fig. 7.1). Si cette identification globale ne nous paraît pas vraisemblable, cet objet très inhabituel n'ayant que peu de rapport, dans son ensemble, avec un linga ou même un caturmukhalinga, son affiliation shivaïte n'en semble pas moins exacte. Pourtant, on ne saurait affirmer qu'il s'agit là de la principale statue de culte de ce sanctuaire, d'autant qu'elle fut retrouvée dans ce qui a été identifié comme le gopura sud du temple ${ }^{23}$ (Lunet de Lajonquière 1907, p. 69).

Les autres fragments de décor sculptés repérables en surface, souvent difficiles à dater ou postérieurs au règne de Yaśovarman ${ }^{\mathrm{I} r}$, n'apportent pas d'informations supplémentaires. Pour ce qui concerne les phases de construction, il faut pourtant mentionner les pilastres du gopura sud, seul décor architectural encore en place, que Nalesini compare au décor des fausses portes de Thommanon (fig. 7.2). La deuxième phase de construction encore observable daterait alors de la fin $\mathrm{du} \mathrm{XI}^{\mathrm{e}}$ ou du XII ${ }^{\mathrm{e}}$ siècle de notre ère (Nalesini 2000, p. 132).

\section{L’āśrama de Vat Phu (l'inscription K. 1005)}

Des deux groupes de fragments de stèles conservés à Vat Phu, seul celui qui est enregistré sous le numéro K. 1005 est le seul à avoir gardé les deux derniers pāda de la stance XXXVI. Nous en livrons ici la lecture inédite :

bhadreśvarāya sa idam śăsanan krtavān iti $\|^{24}$

«... il a fait cet édit pour Bhadreśvara ${ }^{25} . »$

Au Cambodge, plusieurs dieux ont porté le nom de Bhadreśvara ${ }^{26}$ montrant, par là, que cette figure shivaïte occupait au sein du panthéon khmer une place importante. Celui qui fut la divinité principale du lingaparvata, « la montagne qui est un linga», situé à Vat Phu, était sans doute le plus vénéré. Sa célébrité est attestée dès les débuts de la période préangkorienne ${ }^{27}$ et il semble que le sanctuaire de Vat Phu soit devenu très tôt un lieu de pèlerinage de premier ordre. L'ancienneté de ce sanctuaire et son importante sacralité sont sûrement à l'origine de la multiplication des fondations shivaïtes reprenant le nom de Bhadreśvara.

La stèle portant l'inscription K. 1005 n'a pas été retrouvée à Vat Phu même mais à deux kilomètres au sud, pouvant laisser croire que la divinité de l'inscription est celle d'un autre temple. Cependant, la proximité du lieu de découverte et de Vat Phu, associée

23. Ou plus exactement sud-ouest, le temple de Houay Tomo étant nettement désaxé en raison de contraintes topographiques sans doute liées au cours de la rivière qui le borde.

24. Édition fondée sur des clichés communiqués par M. Lorrillard. La stance XXXVI est incomplète sur la face B et le nom de la divinité, en particulier, n'est lisible que sur la face A. Nous suivons ici l'usage adopté par $\mathrm{A}$. Bergaigne et $\mathrm{A}$. Barth, qui veut que $\mathrm{A}$ désigne la face en caractères « étrangers ».

25. À la lumière des éléments rapportés dans la note 15 , il est possible de traduire autrement la stance dans son intégralité : «Après avoir donné le vénérable Yaśodharāśrama à Bhadreśvara en lune (un), un, formes (huit), il a fait cet édit ».

26. Ce nom signifie « le seigneur auspicieux » et désigne Śiva. À propos du succès de cette divinité au Cambodge, cf. Sanderson 2003-2004, p. 240, 410, 418 et Estève 2009. p. 423-431.

27. Les témoignages épigraphiques (cf. Barth 1902, par cxcmple) ont été, depuis, confirmés par les fouilles de M. Santoni qui ont prouvé que les premières installations de ce sanctuaire sont contemporaines de la fondation de la cité à laquelle il était lié. Cette cité était implantée sur les rives du Mékong et son occupation aurait atteint son extension maximale entre le $\mathrm{v}^{\mathrm{c}}$ et le vII siècle de notre ère (Santoni et al. 1997, p. 242-243 : Santoni \& Souksavatdy 1999[b], p. 199). 
à la sacralité hors normes du Bhadreśvara du lingaparvata, rendent très vraisemblables l'association de ce dernier à l'āśrama.

Plusieurs des divinités qui sont mentionnées dans les dédicaces des inscriptions digraphiques apparaissent ailleurs dans le corpus épigraphique khmer, mais les occurrences de ces théonymes se trouvent dans des inscriptions provenant de régions diverses, rendant souvent difficile l'identification du sanctuaire où elles résidaient, d'autant qu'il ne s'agit jamais de divinités aussi célèbres que le Bhadreśvara de Vat Phu ${ }^{28}$.

La dédicace de l'inscription K. 1005 prend alors toute son importance. Elle fournit en effet une information relative à la localisation de ces institutions. Ainsi que le faisait remarquer G. Cœedès, il est vraisemblable que les āśrama provinciaux ont en grande partie été construits en matériaux périssables, comme les grands āśrama d'Angkor, raison pour laquelle ils restent difficiles à localiser $(1932$, p. 112). Plusieurs questions restent pourtant sans réponse. Ces édifices étaient-ils implantés dans le voisinage immédiat des sanctuaires auxquels ils étaient liés, ou en étaient-ils des extensions, sortes de succursales pouvant être très éloignées ? Possédaient-ils quelques infrastructures pérennes ${ }^{29}$ ? Enfin, leur organisation spatiale suivait-elle un modèle constant, comme c'était le cas, semble-t-il, à Angkor ${ }^{30}$ ?

Le fait que l'inscription K. 1005 lie cet āśrama à Bhadreśvara ne permet pas encore de répondre à ces questions, mais oriente déjà de futures recherches. En effet, comme nous l'avons dit, le nom de cette divinité a le mérite, dans cette région, de renvoyer avec certitude au sanctuaire bien connu de Vat Phu, contrairement au nom de Rudrānī dont on ne peut garantir qu'il fut associé dès l'origine à Houay Tomo, puisque l'inscription K. 362 aurait pu être transportée sur ce site après l'abandon de l'áśrama ${ }^{31}$.

Si l'on suit le cas de Vat Phu, en considérant qu'il montre la règle en vigueur pour tous les ermitages, il faut done supposer que les āśrama de Yaśovarman I er étaient liés à des temples dont ils n'étaient pas très éloignés géographiquement. Ceci constitue un indice important pour les recherches archéologiques à Vat Phu - et en l'occurrence, pour la localisation de l'ásirama -, même si la complexité du site et les nombreux aménagements qui lui sont associés rendront l'identification de cet ermitage problématique. Outre les

28. L'inscription K. 42, originaire de Preah Ongkar, dans la province de Kampot, est par exemple dédiée à Śrī Brahmarakșasa, nom que l’on retrouve dans quatre inscriptions provenant de sites éloignés

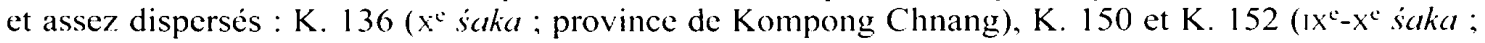
province de Kompong Thom), et K. 669 (894 saka ; province de Preah Vihear).

29. À Angkor, une étude géoradar réalisée en 2010 par Till F. Sonnemann ( $P h D$, University of Sydney) a permis d'établir que l'äśrama de Prasat Komnap sud comprenait un bâtiment long de $25 \mathrm{~m}$ dans sa partie orientale, au sud-ouest de l'édicule à stèle. Sa taille et sa position laissent supposer qu'il s'agit d'un bâtiment équivalent à celui étudié par G. Trouvé à Prei Prasat et à celui qu'il avait signalé au Prasat Komnap nord. Une prospection géoradar a également mis en évidence une structure au même endroit, au Prasat Ong Mong, mais le signal, moins net, ne permet pas d'affirmer qu'elle était de forme et de taille équivalente (Estève \& Soutif 2010, p. 16-24).

30. Sur ce sujet, cf. Pottier 2003 et Estève \& Soutif 2010.

31. C'est malheureusement le cas de plusieurs inscriptions de fondation d'äśrama, à commencer par celle de l'àśrama bouddhique d'Angkor (K. 290). découverte à lep Pranam, mais provenant vraisemblablement du Prasat Ong Mong (Cœè̀s 1932, p. 111), ou encore de la deuxième inscription digraphique de Preah Kô (K. 1223) retrouvée par Ch. Pottier au poste de police de ce site ; elle proviendrait « d"un emplacement plus au Nord, vers le Phnom Bok (?) » (Pottier 2005, p. 479, n. 47). 
structures pérennes visibles sur le site, on sait notamment grâce à d'autres inscriptions que d'autres ermitages ont été fondés sur ce site ${ }^{32}$.

Cette dédicace permet également de formuler l'hypothèse selon laquelle le dieu auquel l'áśrama était dédié ne résidait pas en son sein mais était, au contraire, installé dans un temple préexistant, auquel l'áśrama se référait ${ }^{33}$; ceci conduit à s'interroger directement sur le type d'activité religieuse qui prenait place dans ces institutions. On sait, en effet, que les āśrama d'Angkor accueillaient des divinités ${ }^{34}$ et donc des cérémonies religieuses. Mais dans le cas de l'ermitage de Vat Phu, puisque la divinité à laquelle il était rattaché possédait déjà son propre sanctuaire d'après notre hypothèse, on peut se demander si ces cérémonies religieuses se déroulaient en présence d'un substitut ou d'un double érigé dans l'ermitage, ou simplement en présence d'autres dieux. Il est également possible que la divinité de Vat Phu ait fait l'objet d'une cérémonie particulière lors de la construction de l'āśrama.

Enfin, selon une dernière hypothèse on pourrait supposer que cet āśrama s'apparente simplement à un lieu d'accueil pour une communauté religieuse (impliquant éventuellement un enseignement religieux), c'est-à-dire à une structure dépourvue de lieu de culte proprement dit.

Le cas de Vat Phu incite aussi à considérer que ces dieux faisaient partie des divinités hautement sacrées du pays khmer et donc que les emplacements des áśrama correspondent à autant de lieux particulièrement saints, nous permettant, à présent, d'identifier et de localiser les sanctuaires qui accueillaient ces grands dieux ${ }^{35}$. Les áśrama représentent ainsi des bornes sacrées placées par Yaśovarman $\mathrm{I}^{\text {er }}$ sur un territoire déjà organisé du point de vue religieux; il n'a fait que doter ces lieux saints d'ermitages.

Les questions soulevées par ces institutions ne manquent pas, que ce soit concernant leurs fonctions ou leur apport pour les religions du Cambodge ancien.

\section{Dotation et règle des āśrama}

Si une part importante était réservée, dans ces inscriptions digraphiques, à la glorification du roi, la suite de ces textes, malheureusement plus courte, était entièrement consacrée à la charte régissant les àśrama.

Les stances XXXVII et XXXVIII évoquent d'abord les moyens de subsistance attribués par le roi à l'āśrama afin d'assurer son bon fonctionnement. Leur détail n'est pas

32. La stance VII de l'inscription K. 366 (datée de 1061 śaka; IC V, p. 288), par exemple, rappelle la fondation d'un ermitage sur le site de Vat Phu en 1061 śaka par le vénérable du lieu saint; cet àsrama, pourvu de trois pièces d'eau, se nommait le Bhadreśvarālaya.

33. L'inscription de Preah Kô tend également à confirmer ceci : d'après l'inscription K. 309, la divinité à laquelle l'ermitage est dédié est Parameśā, théonyme qui correspond manifestement à Parameśvara, la divinité principale de Preah Kò (ISCC, p. 300). Le seul autre cas à ce jour serait l'inscription K. 346 de Prasat Neak Buos qui rattache l'ermitage construit au Ganeśa de Candanādri, toponyme que l'on retrouve peut-être sous la forme candanagiri dans l'épigraphie du temple (cf. inscription K. $344, I C$ VI, p. 164). On notera toutefois que ce Ganeśa n'est pas la divinité principale de Neak Buos, celle-ci étant un Śivapāda et ce, depuis l'époque préangkorienne.

34. Des fragments de statuaire, dont plusieurs piédestaux, ont été retrouvés en 1932 par G. Trouvé et Henri Marchal dans la partie orientale de Prei Prasat, Prasat Ong Mong et des Prasat Komnap nord et sud (Trouvé 1932, p. 121 ; $R C A$ octobre 1931, janvier-avril, juin 1932, juin 1933).

35. Au sujet de la sacralité des lieux d’implantation des áśrama, G. Coedès écrivait déjà en 1932 : "L'année même de son avènement en 811 çaka, le roi Yaçovarman I fit placer dans divers lieux saints de ses états des stèles portant chacune sur leurs deux faces la même inscription sanskrite (...) ce sont les inscriptions « digraphiques » dont on connaît actuellement douze répliques » (1932, p. 108). 
donné, comme cela est la règle dans les inscriptions en sanskrit de cette époque. En effet, l'objectif de ces textes est plus d'exalter la prodigalité du donateur que de faire l'inventaire des biens donnés, contrairement aux inscriptions en khmer, plus administratives, qui livrent souvent, de ce fait, d'interminables énumérations.

L'auteur a toutefois veillé à montrer que rien n'avait été laissé au hasard, et que l'āśrama était doté de tout le nécessaire : gemmes, métaux précieux, personnel, bétail et patrimoine foncier. Il n'est malheureusement pas précisé qui avait la charge de gérer ce patrimoine et l'on ignore donc si ces äśrama, affectés à des sanctuaires, conservaient une certaine indépendance.

La suite du texte rapporte, au moins en partie, leur règle. En premier lieu (st. XXXIXXLIV), le texte énumère les prescriptions conditionnant l'accueil des hôtes, qu'ils soient religieux ou non. Il s'agit d'abord de règlements vestimentaires qui, sans être particulièrement austères, imposent une tenue modeste, à l'exception des dignitaires de très haut rang (le roi, les brāhmanes et les fils de roi) qui peuvent conserver leurs parures. Suivent quelques précisions très générales relatives au comportement des hôtes et au droit d'accès. En dehors des serviteurs du roi et de la cour, les hommes du commun ne sont pas acceptés, et une conduite irréprochable est attendue des ascètes accueillis, quelle que soit leur confession. La seule règle plus concrète en matière de comportement est l'obligation de descendre de son char et de ne pas utiliser de parasol aux abords de l'áśrama ${ }^{36}$, ce qui revient, pour les dignitaires, à abandonner leurs principaux attributs ${ }^{37}$.

La deuxième partie de ces prescriptions ne tient plus aux devoirs des hôtes, mais à ceux du personnel de l'äśrama, et en particulier de son vénérable (kulapati). Ce dernier est invité à assurer le gîte et le couvert des hôtes, et à leur rendre les honneurs en fonction de leur position hiérarchique.

Contrairement au texte des stèles de fondation des áśrama de la capitale, beaucoup plus long, où l'exposé des règles occupe plus de cinquante stances, la règle des institutions de province n'est que partiellement reproduite dans ces inscriptions. Bien des aspects de leur fonctionnement n'y sont pas abordés, à commencer par les prescriptions de nourriture et de fournitures qui sont détaillées de façon précise à Angkor, suggérant que, sur ce point, les āśrama suivaient la règle des sanctuaires auxquels ils étaient affectés.

L'élément le plus notable est le fait que ces prescriptions soient communes à toutes les stèles digraphiques quelle que soit la confession du sanctuaire auquel elles étaient associées. Ceci est d'autant plus intéressant que les textes des inscriptions de fondation des äśrama d'Angkor diffèrent précisément entre eux pour ce qui concerne les fournitures accordées annuellement aux ascètes, que l'äśrama soit shivaïte, vishnouite ou bouddhique ${ }^{38}$. Il serait pourtant faux de penser que les ásrama n'accueillaient que les ascètes de la confession de leur sanctuaire ; comme nous l'avons déjà signalé, le texte précise que « les brāhmanes, les sectateurs de Viṣnu ou de Śiva et tous les gens de bien pourront y coucher, y réciter leur prières à voix basse et s'y livrer à la méditation " (st. XLIII ; $I S C C$, p. 368,375 ). Peut-être faut-il supposer que le coût de telles fondations interdisait de les multiplier. La mise à disposition de leurs infrastructures à l'ensemble des confessions qui cohabitaient au Cambodge à cette époque peut alors être interprétée selon des

36. On notera qu'une inscription datant des $v l^{-}-v I^{\circ}$ siècles de notre ère mentionnait déjà ce type de prescription pour le temple de Vat Phu (K. 367, st. V, Barth 1902, p. 238, 240).

37. Au sujet des insignes de dignité, on se reportera par exemple au chapitre 4 des mémoires de Zhou Daguan (Pelliot 1951, p. 13-14).

38. Sur ce sujet, cf. Coedès 1932, p. 106-108 et Estève 2009, p. 338-348. 
motifs économiques, afin de minimiser les coûts, ou selon des motifs religieux, comme exprimant l'« œcuménisme » des comportements religieux du Cambodge.

\section{"Envoi " et paraphe}

Comme c'est souvent le cas dans l'épigraphic khmèrc, après l'édit même, la fin du texte comprend d'abord une stance d'imprécation vouant à l'enfer ceux qui transgresseraient le décret, tant «que dureront le soleil et la lune » (st. XLVIII), suivie d'une bénédiction promettant au contraire la moitié des mérites gagnés par le fondateur à qui protègerait l'āsirama (st. XLIX).

Ce qui est plus rare, c'est que cette conclusion est suivie sur chaque face d'un court texte, respectivement en sanskrit pour la face A (stance $L$ ) et en khmer pour la face B, rédigé dans les deux cas dans les caractères « classiques » du Cambodge ancien, et porteur de la même information :

face A :

amvujendrapratäpena kamvujendrena nirmmitam

amvujäksena tenedam kamvujäksaram äkhyava

«Majestueux comme l'Indra des ambujas (lotus. le soleil) le roi des Kambujas aux yeux d'ambujas (de lotus) a tracé ces caractères nommés caractères des Kambujas. » (ISCC, p. 368.376)

face B :

nệ śloka neh gi man srasir" nu kamvujākșara

"Ce poème est écrit avec les caractères des Kambujas."

La nature même des stèles digraphiques amène d'abord à se demander lequel des deux alphabets utilisés est qualifié de kamvıjākșara. On serait tenté de supposer en premier lieu qu'il s'agit de celui employé pour la face $B$, avec lequel ces deux passages sont rédigés. Originaire d'Inde du Sud, il avait été introduit au Cambodge aux environs du v' siècle de notre ère, et avait évolué jusqu'à prendre une forme, sinon « classique ", en tout cas très régulière, sous le règne d'Indravarman $I^{\text {er }}$ et surtout sous celui de son fils Yaśovarman $I^{\mathrm{er}}$. Pourtant, l'inscription K. 290 semble contredire cette hypothèse. En effet, cette stèle est entièrement rédigée dans le nouvel alphabet que Yaśovarman $I^{\text {er }}$ essayait de diffuser, y compris sa dernière stance qui répète exactement le texte de la stance $\mathrm{L}$ de la face $\mathrm{A}$ des inscriptions digraphiques (Cœdès 1908, p. 214, 225 ; estampage EFEO n 559). Ainsi, à moins de considérer que les deux alphabets étaient désignés comme kamvujajkssara ${ }^{39}$, il faut se résoudre à conclure que ce terme désignait plutôt les caractères de la face $\mathrm{A}$, qu'A. Barth et A. Bergaigne appelaient « caractères étrangers ».

Qualifié souvent à tort de devanāgarī, cet alphabet n'en appartient pas moins à « la classe des alphabets nāgarì du nord » de l'Inde (ISCC, p. 349). Comme l'a déjà souligné Richard Salomon. il s'agit en fait d'une sorte de siddhamätrk $\bar{a}^{+0}$, caractères qui se sont

39. Cette hypothèse était celle de Barth (ISCC, p. 351). Elle nous semble étonnante mais reste envisagcable.

40. Pour un exemple de cet alphabet, on pourra se reporter aux tableaux palćographiques de l'Inde classique (Renou \& Filliozat 2000, p. 690-692) et au document 9 pour ce qui concerne la forme qu iil prit au Cambodge. Des descriptions de cet alphabet ainsi que des comparaisons avec ses équivalents javanais et indiens sont présentées par A. Barth (ISCC. p. 347-354) et J. de Casparis (1979, p. 394. 
développés en Inde entre la fin $\mathrm{du} \mathrm{VI}^{\mathrm{e}}$ et le $\mathrm{x}^{\mathrm{c}}$ siècle de notre ère et se caractérisent par un aspect très anguleux $(1998$, p. 39, 156). Au Cambodge, ils sont, de plus, particulièrement ornés de fleurons.

Barth avait remarqué qu'aux alentours des $\mathrm{VII}^{\mathrm{e}}$-VIII $\mathrm{C}^{\mathrm{C}}$ siècles de notre ère, un alphabet de même origine avait également été introduit en Inde du Sud et à Java (ISCC, p. 349-351). À propos du succès de la siddhamātrkā et de sa transmission, R. Salomon relève d'ailleurs qu'en Inde, il préfigure en cela le rôle de la devanāgarī comme écriture quasi nationale, parfois préférée aux écritures locales pour écrire le sanskrit (1998, p. 39). Bien que l'on ignore encore les circonstances de son introduction au Cambodge, celle-ci n'a donc rien d'étonnant quand on sait l'importance des contacts que ce royaume entretenait tant avec l'Inde qu'avec Java. Qu'il s'agisse d'un simple phénomène de mode ou d'une nécessité liée à ces réseaux internationaux, il semble en tout cas que la diffusion de cet alphabet tenait particulièrement à cœur à Yaśovarman $I^{\mathrm{er}}$ et il est intéressant de noter sur ce sujet que, dans l'inscription de fondation de Lolei, l'habileté du roi pour les écritures (lipi) est soulignée dans son éloge, parmi d'autres mérites (K. 323, st. LI ; ISCC, p. 407, n. 3). Quoi qu'il en soit, cette volonté de diffusion de la nouvelle graphie est particulièrement notable dans le fait que ces inscriptions digraphiques, parce qu'elles étaient disséminées dans tout le territoire contrôlé par le roi, fournissaient un outil didactique pour son apprentissage. Le contraste est d'autant plus marquant qu'à Angkor, les inscriptions de fondation d'áśrama étaient entièrement en caractères " étrangers ", qui devaient donc déjà être maîtrisés par les savants de la capitale. Ces inscriptions digraphiques constituent ainsi un document riche d'enseignements pour l'histoire culturelle, montrant les méthodes employées par un roi pour, à la fois, étendre son emprise jusqu'aux marches de l'empire et éduquer les populations. On notera cependant que cette tentative d'introduction a manifestement échoué, puisque l'usage de ces caractères se limite apparemment à ce règne.

Le fait d'utiliser sur les deux faces, pour cette conclusion, les caractères khmers «classiques », et ce, en khmer comme en sanskrit, témoigne encore de cette volonté de transmission. Sur la face $\mathrm{A}$, la stance $\mathrm{L}$ constitue même une sorte de signature qui rappelle qu'il s'agit bien là d'une volonté royale. Ainsi, après avoir affirmé sa légitimité par sa naissance et ses mérites, puis édicté ses volontés, le roi réaffirme, par ce «paraphe », la paternité de cet édit tout en cautionnant l'usage d'une nouvelle graphie.

\section{Post-scriptum}

À propos de l'inscription K. 362 de Houay Tomo, A. Bergaigne notait : « B compte 38 lignes, dont la dernière est occupée par la clause en langue khmère séparée du texte par une rosace, et puis, après un simple signe de ponctuation, par un śloka anushtubh en caractères plus petits et moins profondément gravés, qui est particulier au texte B de ce numéro » (ISCC, p. 390). C'était là une spécificité de cette stèle par rapport à toutes les autres stèles digraphiques connues alors, que l'unicité de l'occurrence empêchait d'interpréter. Or, l'importance de cette cinquantième stance de la face B est aujourd'hui amplifiée par un fait nouveau : bien qu'incomplet, ce même śloka se retrouve à la dernière ligne de la face $\mathrm{B}$ de la stèle digraphique de Vat Phu K. 1005, inédite à ce jour :

n. 43). Ce dernier a également discuté de l’importance de l'étude de ces alphabets pour la connaissance des relations entre l'Inde et l'Asic du Sud-Est (op. cit., p. 390-391 et 1982, p. 132-138). 


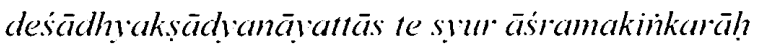

paratanträh kulapatau [täpase ceti sáasanam $\left.\left.\right|_{i}\right]^{+1}$

"Que les serviteurs du couvent ne soient pas mis en réquisition par le gouverneur de la province et les autres fonctionnaires, et qu'ils soient (uniquement) aux ordres du chef de la communauté et des religieux. Tel est (notre) commandement. » (ibid.)

Dans l'inscription K. 1005, également, la gravure présente des différences : plus petite, plus ramassée et moins profondément gravée (fig. 10), même si on a l'impression qu'une même main a œuvré pour l'ensemble du texte, incitant à penser que ce dernier śloka est probablement contemporain du reste de l'inscription. Les deux inscriptions du Laos font donc preuve d'une même spécificité, une stance finale, identique dans son contenu et dans son esthétique, rajoutée en fin de texte. Faut-il en conclure que des événements particuliers avaient eu lieu dans la région, le gouverneur de la province ayant une tendance à s'accaparer la force de travail de l'ermitage ? Ou s'agit-il d'une disposition dont la particularité repose sur la situation géographique de cette frange nord-est de l'empire? Cette dernière hypothèse implique que cette région aurait été moins assujettie au pouvoir de la capitale que les autres ou, plus récemment, et qu'elle ne connaissait pas les us et coutumes khmers concernant la gestion des biens religieux. Ce genre de prescription peut se trouver dans des inscriptions du pays khmer, où elle est formulée avec le même type de vocabulaire ; ici seule la disparité par rapport aux autres inscriptions digraphiques peut nous aider à formuler des hypothèses.

Il en ressort avant tout une affirmation supplémentaire de la volonté du souverain d'imposer son autorité jusque dans ce qui fut sa province la plus septentrionale, et une confirmation du fait que cette emprise du roi s'exprime par le vecteur religieux que sont les ermitages. La conclusion obvie de cette stance supplémentaire est l'entérinement de la protection de la communauté religieuse vis-à-vis du pouvoir politique local.

\section{Deux āśrama dans la région de Vat Phu}

Nous avons évoqué à plusieurs reprises les deux groupes de fragments de stèles digraphiques inédites conservés dans les réserves du musée de Vat Phu; il reste à présent à les étudier plus en détail, en particulier celui qui n'avait pas été inventorié par G. Coedès.

Le premier groupe correspond à la partie inférieure d'une stèle en grès gris. Il comprend deux fragments mesurant respectivement $82 \times 63 \times 11 \mathrm{~cm}$ et $50 \times 34 \times 11 \mathrm{~cm}$. Ils sont conservés sous les numéros VP I 149-1 et VP I 149-2. Les deux blocs sont jointifs et l'ensemble mesure $82 \times 77,5 \times 11 \mathrm{~cm}^{42}$. En dépit de délitements ayant entraîné la perte de nombreux caractères, il semble que nous disposions de toute la largeur de la stèle. En tout, dix-neuf lignes sont partiellement conservées sur la face A et vingt-trois sur la face B. Nous manquons de renseignements précis pour ce qui concerne les circonstances de la découverte de ces fragments, en dehors du fait qu'ils furent trouvés à $2 \mathrm{~km}$ au sud de Vat Phu, probablement dans les années 1960, puisque cette inscription fut la dernière inventoriée par G. Cœdès, sous le numéro K. 1005.

Nous possédons encore moins d'éléments pour ce qui concerne le second groupe de fragments, conservés sous les numéros VP I 452-1 à VP I 452-3. Il semble que leur découverte soit assez récente et qu'ils proviennent également des environs du site de

41. La partie entre crochets est détruite et restituée à partir du texte de l'inscription K. 362 .

42. Les dimensions sont données dans cet ordre : hauteur, largeur puis épaisseur (Christine Hawixbrock et David Bazin, comm. pers.). 
Vat Phu. Il s'agit cette fois de trois morceaux de la partie supérieure d'une stèle sculptée dans le même matériau que K. 1005. Ils mesurent respectivement $51 \times 39 \times 10,5 \mathrm{~cm}$, $33 \times 24 \times 10,5 \mathrm{~cm}$ et $18 \times 18 \times 10,5 \mathrm{~cm}$, et l'ensemble environ $60 \times 57 \times 10,5 \mathrm{~cm}$. Étant donné les dimensions des parties conservées, on peut estimer la largeur de la stèle à environ $75 \mathrm{~cm}$. Si l'on se base sur la face B, les deux fragments les plus importants correspondent aux parties gauche et centrale de la moitié supérieure. Le troisième vient manifestement se placer dans la partie inférieure gauche, mais n'est jointif avec aucun des deux autres. Seule la lecture permet donc de le positionner (Michel Lorrillard, comm. pers.). Une fois ces fragments rassemblés, on peut relever les restes de seize lignes sur chaque face.

La complémentarité physique de ces deux groupes de fragments, la similarité de leur matériau et le fait que leurs dimensions coïncident ${ }^{43}$ sont autant d'éléments incitant à penser qu'ils appartiennent en fait à une même stèle.

La lecture du texte a permis de confirmer ce que ces indices permettaient de présumer : les deux ensembles de fragments ne forment qu'une seule et même stèle digraphique. La comparaison avec l'édition publiée par A. Bergaigne des parties conservées sur les deux faces de tous les fragments permet de constater que le texte est manifestement conforme à celui des autres inscriptions digraphiques connues, mais aussi qu'il s'agit d'une inscription digraphique inédite ${ }^{44}$. Enfin, il n'existe aucun recoupement entre les textes des dcux groupcs dc fragments conservés au musée de Vat Phu. La meilleure preuve de cette unicité se manifeste dans les deux derniers pāda de la stance XVI de la face B lorsque sont apposés les fragments idoines provenant des deux ensembles (fig. 11$)^{45}$ :

[bhūbhṛtputryām iva purabhidotpāditah] $k a \bar{r} \cdot t[t] i K \mathfrak{Y} \boldsymbol{y} \boldsymbol{a}-$

[ś] śa|k|t|im| v|i|bhrad r[i]puku[labhidaṃ śrīyaśovarmmadevah ||]

Les textes se rejoignent au niveau de la syllabe $K e$, le $k$ étant partiellement lisible sur les deux fragments. Il s'agit là du point de contact le plus évident entre les parties inférieure et supérieure de la stèle. Il faut donc désormais considérer que tous les fragments conservés dans les réserves du musée de Vat Phu appartiennent à l'inscription inventoriée par G. Coedès sous le numéro K. 1005. Cette hypothèse a d'ailleurs été vérifiée au musée de Vat Phu où les cinq fragments ont pu être rassemblés (fig. 12). La stèle, une fois reconstituée, mesure $120 \times 77,5 \times 11 \mathrm{~cm}$. Elle est encore incomplète, mais en tenant compte des lignes communes, trente-quatre lignes sont en partie conservées sur la face $\mathrm{A}$ et trente-huit sur la face B.

Ainsi, deux et non trois stèles de fondation d'āśrama de Yaśovarman I ${ }^{\text {er }}$ sont connues, à ce jour, au Laos, dans la région de Champassak. En admettant que l'äśrama de Rudrāṇī ait été implanté dans le voisinage de Houay Tomo, les deux fondations n'étaient alors

43. Ce détail n'est en effet pas anodin quand on sait que, bien que les stèles digraphiques aient quelques caractéristiques communes, en particulicr l'accolade supérieure tronquée, elles n'ont en revanche pas les mêmes dimensions. L'inscription de Houay Tomo est par exemple d'une largeur équivalente $(74,5 \mathrm{~cm})$, mais épaisse de $15,5 \mathrm{~cm}$. La légère différence constatée entre les fragments des parties inférieure et supérieure $(0,5 \mathrm{~cm})$ n'est pas étonnante. la partic inférieure des stèles étant souvent légèrement plus épaisse.

44. Le fait que la « mise en pierre » soit différente pour chaque stèle digraphique permet de garantir aisément le caractère inédit de nouvelles stèles, même quand la stance XXXVI n'est pas conservée.

45. La typographie choisie pour cette stance est à comprendre comme suit : nous notons entre crochets les parties détruites et restitués à partir de l'édition de référence de l’inscription K. 95 (ISCC, p. 365): les caractères partiellement conservés sur les fragments de la partie supérieure de la stèle sont notés en italique et ceux de la partie inféricure en gras. Enfin, le seul caractère qui soit en partie visible sur deux blocs appartenant aux deux groupes de fragments est noté en majuscule. 
éloignées que d'une douzaine de kilomètres à vol d'oiseau, une proximité un peu surprenante au premier abord. Au sujet de la fondation de ces deux ä́ŕrama, O. Nalesini note :

" [it] intended to recognise the almost equal rank of the two places, preserving the memory of their mutual connections in the religious representation: in fact, the dedication of the ásrama to the sákti of Śiva (Rudrānī) linked Huei Thamô to Wat Phu as strongly as the two halves of a pair, and extended the protection of a powerful warrior goddess over a site perceived as dangerously located for it stood on the boundary between the ordered Khmer world and the chaos of the forests peopled by the savages. » $(2000$, p. 133)

Considérer Rudrānī comme la parèdre du Bhadreśvara de Vat Phu, installée sous une forme terrible pour protéger une implantation khmère en territoire hostile, nous paraît assez osé en l'état actuel des connaissances concernant cette déesse, que ce soit en pays indien ou en pays khmer ${ }^{46}$. Il nous semble déjà difficile de considérer le site de Houay Tomo comme isolé et potentiellement dangereux, tant ce que l'on sait de la situation aux marches de l'empire et des relations qu'entretenaient les Khmers avec les « sauvages » est limité. Seules des fouilles archéologiques nous permettront peut-être de comprendre pourquoi un àsrama était installé à Houay Tomo, en nous renseignant en particulier sur l'occupation du site, tant à l'époque préangkorienne qu'à la fin du $I x^{*}$ siècle.

Les critères d'élection des sites d'implantation des áśrama de Yaśovarman I ${ }^{\mathrm{er}}$ ne sont pas encore établis avec certitude, mais l'hypothèse de G. Coedès selon laquelle ces fondations étaient attachées à des sanctuaires préexistants probablement importants, trouve un premier élément de confirmation dans la dédicace de l'inscription K. 1005 en faveur de Bhadreśvara (1932, p. 108). L'antériorité du sanctuaire est ainsi prouvée à Vat Phu, et vraisemblable dans les autres cas. Si cette hypothèse se vérifiait, nous disposerions d'un outil idéal pour la constitution d'une cartographie religieuse du Cambodge du $I X^{\mathrm{C}}$ siècle permettant d'étudier la dissémination et l'importance relative des différents courants religieux d'origine indienne qui y étaient implantés.

Cependant, l'importance des sites de Vat Phu et de Houay Tomo n'explique peut-être pas à elle seule la raison de la proximité géographique des āśrama qui leur sont affiliés. En effet, il faut songer que ces lieux étaient également des gîtes d'étapes, susceptibles d'être utilisés par le roi. Or, Vat Phu et Houay Tomo sont séparés par un obstacle de taille, le Mékong, qui est large de près d'un kilomètre et demi à cet endroit. Il est donc possible que l'installation de gîtes d'étapes de part et d'autre de cette frontière naturelle ait répondu à un choix stratégique destiné à faciliter les déplacements entre les différentes

46. L’inscription K. 366 évoque l'installation de plusieurs divinités à Vat Phu, dont une Bhagavatī tuant Mahīṣāsura en 1050 śaka, fait qui pourrait être compris comme alimentant l'hypothèse de l'implantation d'une déesse féroce défendant le territoire à Houay Tomo. Cependant, d’autres Mahīṣāsuramardin̄i se rencontrent dans l'épigraphie et elle ne sont pas, dans ces occurrences, valorisées pour leur aspect féroce ou protecteur. Par exemple, dans l'inscription de Prasat Car K. 257 (901 ścaka, IC IV, p. 140, cf. 1. 29-31), elle est expressément présentée comme " le siège de la défunte reine (kanlon kamrateri añ Añve Danle » une divinité très présente dans le corpus épigraphique et qui ne prend pas habituellement cette forme, et dans l'inscription K. 826 (803 saka. IC I. p. 35), elle est une fondation des « nobles femmes du gynécée, toutes ensemble ». Par aillcurs, les autres occurrences de Rudrānī dans l'épigraphie ne fournissent pas d'éléments que ce soit concernant la nature de cette divinité ou ses caractéristiques : elles désignent soit des anthroponymes de membres de la famille royale (K. 382, K. 289) ou de haut rang (K. 61, K. 79, K. 989. K. 1238), soit des anthroponymes d'esclaves (K. 235). Enfin, dans l'inscription K. 875 (st. IV, Coedes 1937, p. 120), qui est la plus ancienne de ce corpus d'occurrences (ve-v1" siècle de notre ère), Rudrāṇī apparaît dans une stance d invocation en tant. simplèment, que parèdre de Śiva. Cette interprétation de la déesse nous semble être la plus probable. 
provinces du royaume. Leur localisation pourra alors nous renseigner sur le réseau des voies de communication qui sillonnaient le territoire contrôlé par Yaśovarman I ${ }^{\mathrm{er}}$.

Enfin, en plus d'être une frontière naturelle, il est possible que le fleuve ait également matérialisé la frontière de deux circonscriptions administratives. O. Nalesini note que le nombre d'āśrama annoncé dans les inscriptions de Yaśovarman I ${ }^{\mathrm{er}}$, une centaine, correspondait assez bien aux quatre-vingt dix provinces qui composaient l'empire khmer lors du séjour de Zhou Daguan (2000, p. 133 ; Pelliot 1951, p. 32). Naturellement, l'empire en pleine constitution de Yaśovarman $I^{\text {er }} n$ 'avait sans doute que peu de rapports avec celui que visita cet ambassadeur à la fin du XıII siècle, et le nombre de provinces qu'il comportait alors nous est encore inconnu. Par ailleurs, le texte de la stance $L$ de la face B commune à K. 362 et K. 1005 laisserait plutôt supposer que ces deux āśrama appartenaient à la même province. Il reste pourtant envisageable que ce chiffre de cent, idéalisé ou non, renvoie à un autre découpage administratif que celui constaté par Zhou Daguan, où un áśrama dépendant du sanctuaire le plus important de chaque division aurait été construit. Ce ne sont que des hypothèses, mais à terme, c'est donc aussi la carte politique de cette époque que l'étude des âśrama de Yaśovarman I Ir est susceptible de documenter.

Ainsi, à la fois symboles de l'étendue du pouvoir royal et de sa volonté centralisatrice, les āśrama peuvent également être compris comme des vecteurs privilégiés de sa transmission. Ils délimitent le territoire khmer en s'imposant comme marqueurs géographiques et ils apportent la parole du roi jusque dans les franges de l'empire (cf. stance L des inscriptions K. 362 et K. 1005). Ils sont le biais par lequel le roi entend non seulement propager sa renommée mais également étendre la culture khmère : en utilisant la digraphie pour introduire ce nouvel alphabet, Yaśovarman en fait des outils culturels. Ils s'affirment également comme de précieux outils de développement pour la gestion du territoire, puisqu'ils le ponctuaient de nombreux lieux d'accueil. Ils sont enfin des bornes sacrées nous permettant de reconnaître l'importance des sanctuaires auxquels ils étaient associés et donc d'identifier les divinités les plus sacrées de cette époque. Le savoir qu'ils recèlent, sur le plan religieux, est considérable, que ce soit du point de vue de l'organisation rituelle de ces institutions, de l'affiliation sectaire des divinités ou encore des modalités de l' « œuménisme » en vigueur dans les ermitages de province. Et ces connaissances, fondamentales pour cette fin du $I X^{\circ}$ siècle, sont grosses de répercussions pour les siècles suivants : en affirmant la sacralité de lieux bien particuliers, la fondation des áśrama contribue à expliquer la prépondérance de certaines divinités que l'on constate par la suite. En cela, ces fondations nous permettent à la fois de mieux appréhender la hiérarchisation des dieux du Cambodge ancien mais également de faire avancer nos connaissances générales sur les religions en présence.

Même en supposant que les cent āśrama n'aient pas tous été fondés, ces institutions ont manifestement marqué profondément le règne de Yaśovarman $I^{\text {er }}$. Leur étude nous paraît donc fondamentale pour qui souhaite mieux connaître le Cambodge du IX siècle, moment-clé de la fondation de l'empire angkorien. Ils constituent un sujet de recherche fondamental pour l'histoire politique, religieuse et culturelle du Cambodge. 


\section{BIBLIOGRAPHIE}

Abréviations utilisées

APK : Articles sur le pays khmer; cf. Cadès 1989-1992.

BEFEO : Bulletin de l'École française d'Extrême-Orient.

BSEI : Bulletin de la société des études indochinoises.

IC : Inscriptions du Cambodge; cf. C(F.DĖs 1937-1966.

ISCC: Inscriptions sanscrites de Campā et du Cambodge ; cf. BERGiAlG.NE 1893.

$J A:$ Journal asiatique.

NIC : Nouvelles inscriptions du Cambodge; cf. Pol 2001.

RCA : Rapports de la Conservation d'Angkor.

AYMONIER, Étienne

1900-04 Le Cambodge, 3 vol., Paris, Ernest Leroux.

Barth, Auguste

1902 « Stèle de Vat Phou près de Bassac (Laos) », BEFEO 2/3, p. 235-240.

Bhattacharya, Kamaleswar

1961 Les religions brahmaniques dans l'ancien Cambodge d'après l'épigraphie et l'iconographie, Paris, EFEO.

Bergaigne, Abel

1893 Inscriptions sanscrites de Campā et du Cambodge (extraits des notices et extraits des manuscrits de la Bibliothèque nationale $27,2^{\circ}$ partie, $2^{\mathrm{e}}$ fascicule), Paris, Imprimerie nationale, p. 181-632.

BOISSELIER, Jean

1966 Le Cambodge [Manuel d'archéologie d'Extrême-Orient, première partie : Asie du Sud-Est, tome I], Paris, A. et J. Picard.

Bruguier, Bruno

1998 Bibliographie du Cambodge ancien, 2 vol., Paris, EFEO.

CádĖs, George

1908 « La stèle de Tép Pranam (Cambodge) », JA 10/11, p. 203-225.

1932 «Études Cambodgiennes : 30. À la recherche du Yaçodharāçrama », BEFEO 32/1, p. 84-112 [réimp. 1989, APK 1, p. 240-268].

1937 «A new inscription from Fu-nan », Journal of the Greater Indian Society 4 /2, p. $117-121$.

1937-66 Inscriptions du Cambodge, 8 vol., Hanoi et Paris, EFEO.

1989-92 Articles sur le pays khmer, 2 vol., Paris, EFEO, [réimp. des articles sur le Cambodge parus dans le $B E F E O$ et dans les $C E F E O]$.

DE CASPARIS, Johannes

1979 «Palaeography as an Auxiliary Discipline in Research on Early South East Asia », dans R. B. Smith \& W. Watson (eds.), Early South East Asia, Oxford, Oxford University Press, p. 380-394.

1982 « Some Aspects of the Expansion of Nāgarī Script in South and South East Asia », dans G. de la Lama (ed.), South Asia 1, Mexico, El Colegio de México, p. 123-138. 
Estìvı:, Julia

2009 «Étude critique des phénomènes de syncrétisme religieux dans le Cambodge angkorien », thèse de doctorat sous la direction de Gerdi Gerschheimer, École pratique des hautes études, Paris.

Estevi:, Julia \& Dominique SoutıF

2010 Rapport annuel de la mission Yaśodharāśrama, Siem Reap. APSARA/MAE/ EFEO.

FILLIOZAT, Jean

1981 «Sur le śivaïsme et le bouddhisme au Cambodge », BEFEO 70, p. 59-99.

Gaginfux, Pierre-Marie

1977 Recueil des inscriptions du Laos, vol. 1, service des monuments historiques, Vientiane (document ronéotypé).

LUNI:T DI: LA.JONQUIERE: Étienne

1902-11 Inventaire descriptif des monuments du Cambodge, 3 vol., Paris, Ernest Leroux.

NALI:SINI, Oscar

2000 "The Sanctuary of Huei Thamo, and the Historical Problems Raised by its Survey ", dans W. Lobo \& S. Reinemann (eds.), Southeast Asian Archaeology 1998: Proceedings of the 7th Conference of the European Association of Southeast Asian Archaeologists, Hull, Centre for Southeast Asian Studies and Staatliche Museen zu Berlin.

Parmentiler, Henri

1913 «Complément à l'inventaire descriptif des monuments du Cambodge », BEFEO 13/1, p. 1-64.

1939 L'Art khmèr classique. Monuments du quadrant Nord-Est, Paris, EFEO.

Plilliot, Paul

1951 Cf. Zhou Daguan.

PotTll:R, Christophe

2003 «About Yaçovarman's Buddhist açrama in Angkor », dans P. Pichard \& F. Lagirarde (eds.), The Buddhist Monastery: A cross-cultural survey, Paris, EFEO, p. 199-208.

POTTI:R, Christophe \& Rodolfo LUAN-LUNSFORD LUNSFORD

2005 « De brique et de grès : précisions sur les tours de Prah Kô », BEFEO 92, p. 457-495.

Pot, Saveros

2001 Nouvelles inscriptions du Cambodge, t. 2 \& 3 (en un seul volume), Paris, EFEO.

2004 Dictionnaire vieux khmer-firançais-anglais. An Old Khmer-French-English Dictionary. Vacanānukram khmaer cas'-paramn-angles, Paris, L'Harmattan, $2^{c}$ édition [ $1^{\mathrm{re}}$ éd. $1992+$ supplément].

RI:NOL; Louis \& Jean FILLII)ZAT

2000 L'Inde classique. Manuel des études indiennes, t. 2, avec le concours de Paul Demiéville, Olivier Lacombe, Pierre Meile, Paris, EFEO [1 $1^{\text {rc }}$ édition 1953]. 
SALOMON, Richard

1998 Indian Epigraphy: A Guide to the Study of Inscriptions in Sanskrit, Prakrit, and the Other Indo-Aryan Languages, New York, Oxford University Press.

SANDERSON, Alexis

2003-04 "The Śaiva Religion among the Khmers (Part I) », BEFEO 90-91, p. $349-462$.

SANTONI, Marielle et al.

1997 "Excavation at Champasak and Wat Phu (Southern Laos) », R. Ciarla, F. Rispoli (ed.), South-East Asian Archaeology 1992, Rome, p. 233-263.

Santoni, Marielle \& Viengkèo Souksavatdy

1999[a]« L'importance de la recherche archéologique pour la préservation du patrimoine : l'exemple du site de Vat Phu (province de Champasak, sud du Laos) », dans F. Bizot (éd.), Conservation du patrimoine artistique et historique du Laos, Vientiane, EFEO, p. 167-200.

1999[b] « Fouilles sur le site de Vat Phou - Champassak », dans F. Bizot (éd.), Laos Restaurer et préserver le patrimoine national, éditions des Cahiers de France, p. 167-200.

Soltif, Dominique

2009 "Organisation religieuse et profane du temple khmer du VII" au XII" siècle ", thèse de doctorat sous la direction de Michel Jacq-Hergoualc'h, université Paris III-Sorbonne nouvelle.

Trouvi:, Georges

1932 «Etude sur le Prei Pràsàt, le Pràsàt Komnap et l'édicule qui abritait la cinquième stèle inscrite du Baray Oriental », BEFEO 32/1, p. 113-126.

Ziou Daguan

1951 Mémoires sur les coutumes du Cambodge de Tcheou Ta-Kouan [traduites et annotées par Paul Pelliot], Paris, Adrien Maisonneuve. 


\section{ILLUSTRATIONS}

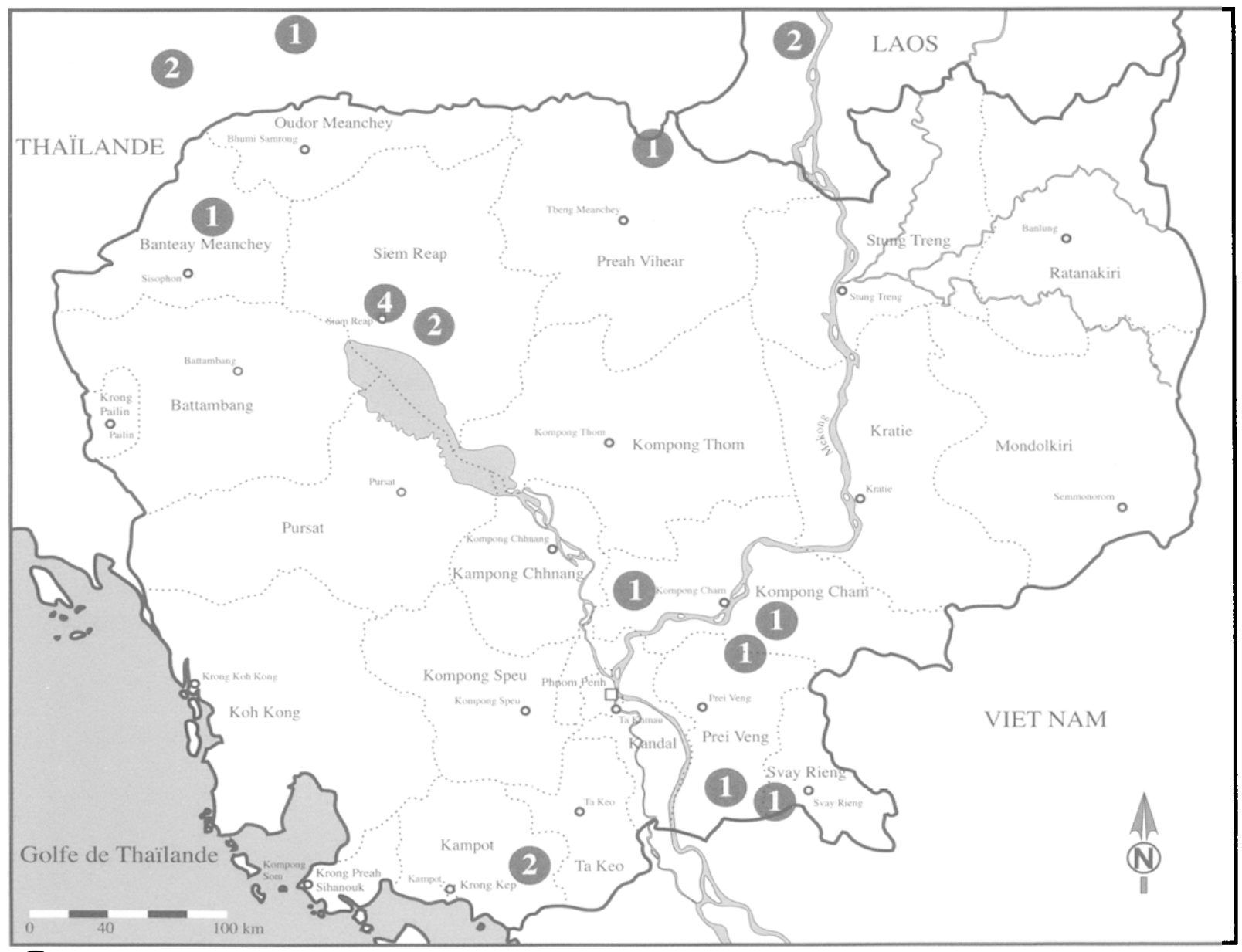

x Nombre d'inscription(s) commémorant la fondation d'un äśrama

Fig. 1 : carte de localisation des inscriptions digraphicques de Yaśovarman IFr. 


\section{Stance XXXVI. ab}

yaśodharāśrame datte śrīmatīndvekamūrttibhih

«Le vénérable Yaśodharāśrama ayant été donné (ici) en lune, un, formes (811 śaka), $\cdots$ »

\begin{tabular}{|c|c|c|c|}
\hline $\mathrm{K}$. & Stance XXXVI. cd & Divinité & Références \\
\hline K. 42 & śrīvrahmarakșase so smai śāsanan kṛtavān iti \|| & Śrī Brahmarakșasa & ISCC, p. 387 \\
\hline K. 45 & Stance manquante & - & ISCC, p. 388 \\
\hline K. 47 & asmai nārāyanāyedaṃ śāsanaṃ sa vyadhād iti \|| & Nārāyaṇa & $I S C C$, p. 386 \\
\hline K. 57 & idam śrīkārttikeyāya śāsanaṃ sa vyadhād iti \|| & Śrī Kārttikeya & $I S C C$, p. 385 \\
\hline K. 95 & candanādrigaṇeśāya śāsanaṃ sa vyadhād idam || & $\begin{array}{l}\text { Ganeśa de Candanādri (Mont } \\
\text { du Santal) }\end{array}$ & $I S C C$, p. 355 \\
\hline K. 101 & pañcalingeśvarāyedaṃ śāsanaṃ sa vyadhād iti \|| & le seigneur aux cinq linga & ISCC, p. 382 \\
\hline K. 110 & śrīraudraparvvateśāya śāsanaṃ sa vyadhād idam \| & le seigneur du Raudraparvata & ISCC, p. 384 \\
\hline K. 223 & idam asyai sa nidrāyai śāsanañ kṛtavān iti $\|$ & Nidrā & $I S C C$, p. 376 \\
\hline K. 309 & idam sa parameśāya śāsanan̉ kṛtavān iti \| & Parameśā & $I S C C$, p. 377 \\
\hline K. 346 & candanādrigaṇeśāya śāsanaṃ sa vyadhād idam \| & $\begin{array}{l}\text { Gaṇeśa de Candanādri (Mont } \\
\text { du Santal) }\end{array}$ & $I S C C$, p. 378 \\
\hline K. 362 & idam asyai sa rudrāṇyai śāsanañ kṛtavān iti \|| & Rudrāṇ̄̄ & $I S C C$, p. 389 \\
\hline K. 479 & Pāda manquants & - & Inédite \\
\hline K. 1005 & bhadreśvarā yas idạ̣ śāsanañ kṛtavān iti || & Bhadreśvara & Inédite \\
\hline K. 1092 & $P \bar{a} d a$ manquants & - & Inédite \\
\hline K. 1093 & Pāda manquants & - & Inédite \\
\hline K. 1223 & Pāda manquants & - & Inédite \\
\hline
\end{tabular}

Fig. 2 : edition synoptique de la stance XXXVI des inscriptions digraphiques de Yaśovarman Itr.

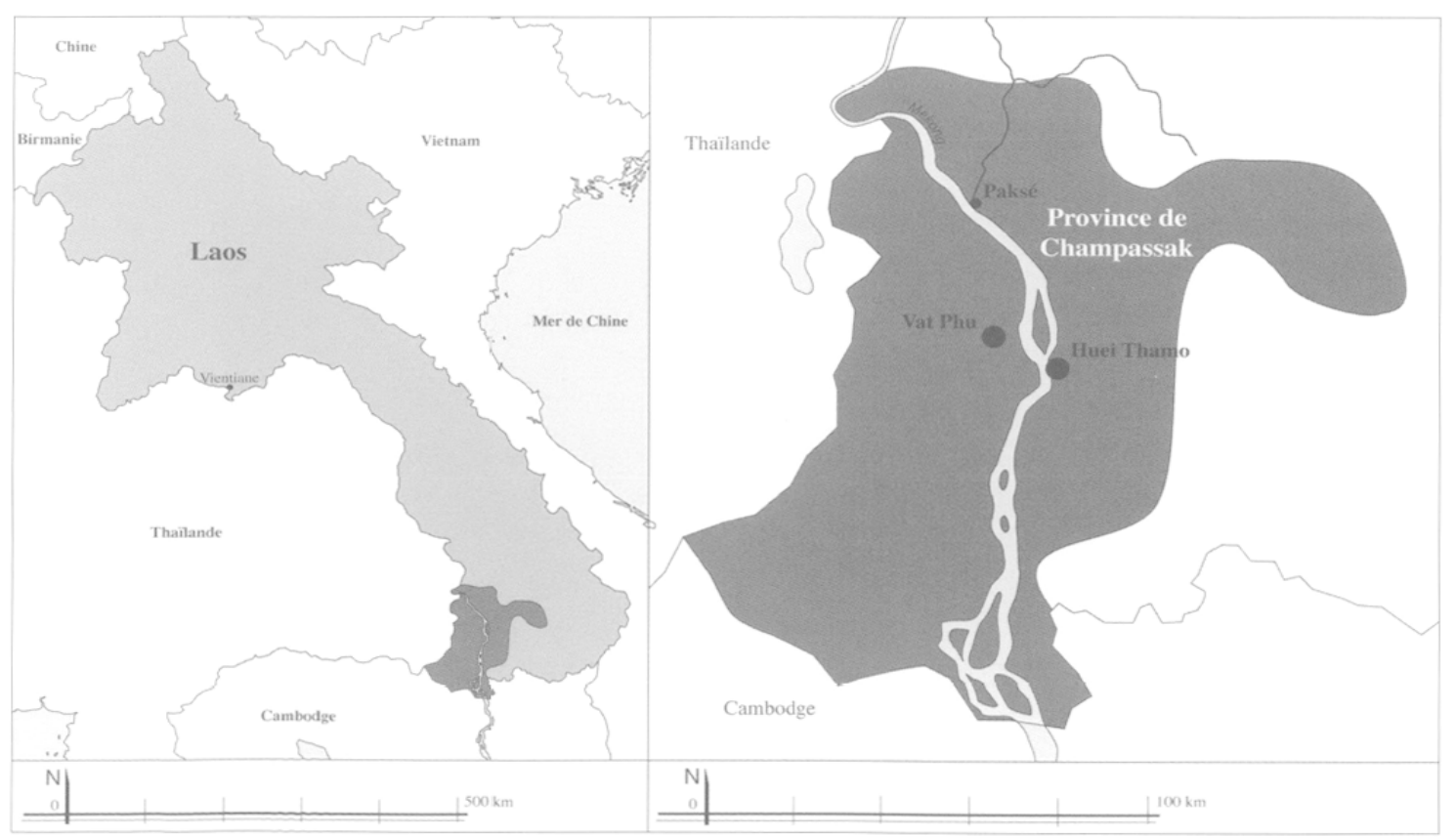

Fig. 3 : cartes de localisation de la province de Champassak et des sites de découverte d’inscriptions digraphiques : Vat Phu et Houay Tomo (vectorisation : Pierre Pichard \& Dominique Soutif). 


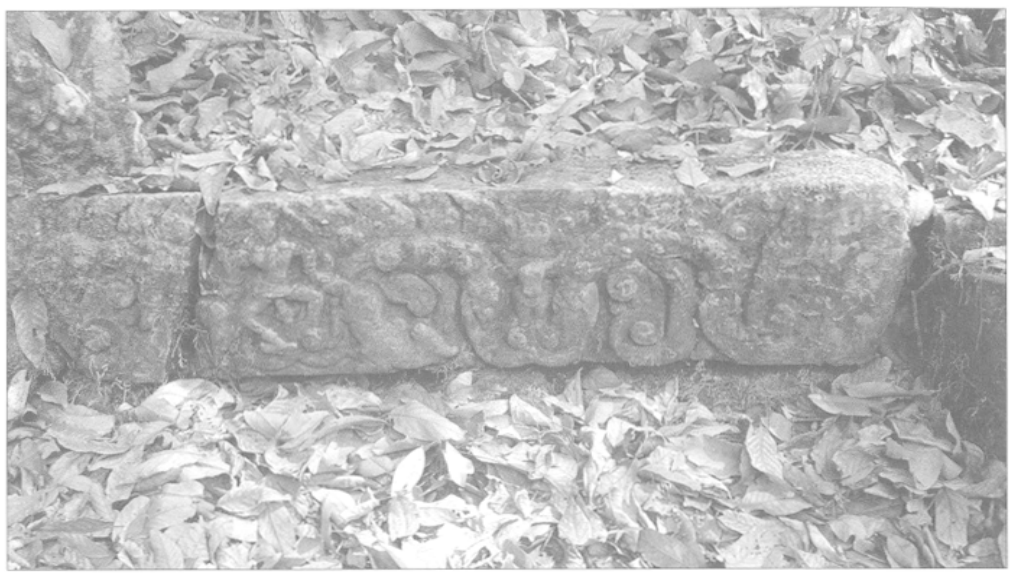

Fig. 4 : linteau décoratif découvert à Houay Tomo : grès : fin du Ix" siècle de notre ère (cliché : Dominique Soutif).

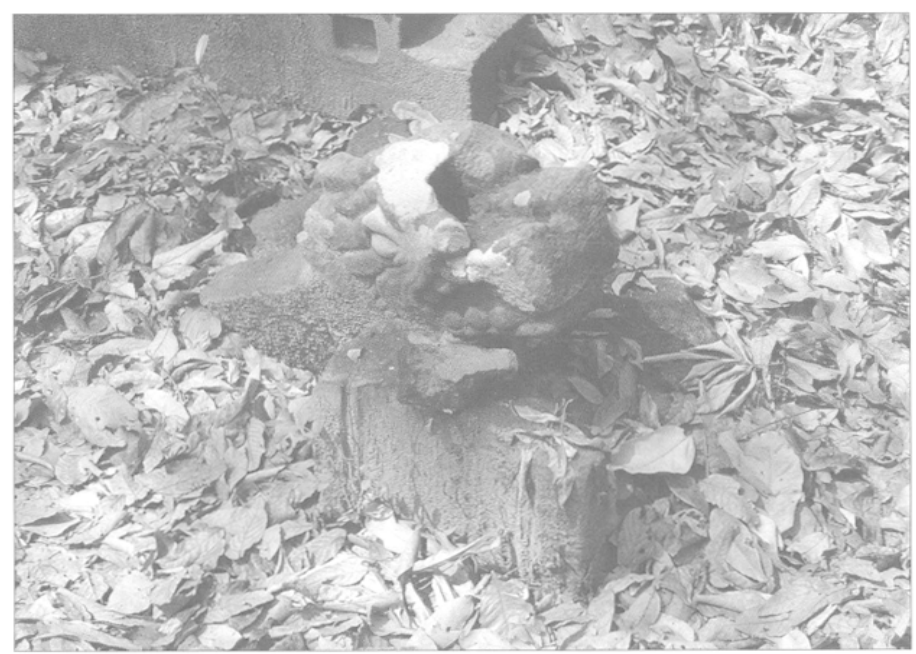

Fig. 5 : canal d’évacuation des eaux lustrales découvert à Houay Tomo ; grès : période préangkorienne (cliché : Dominique Soutif).

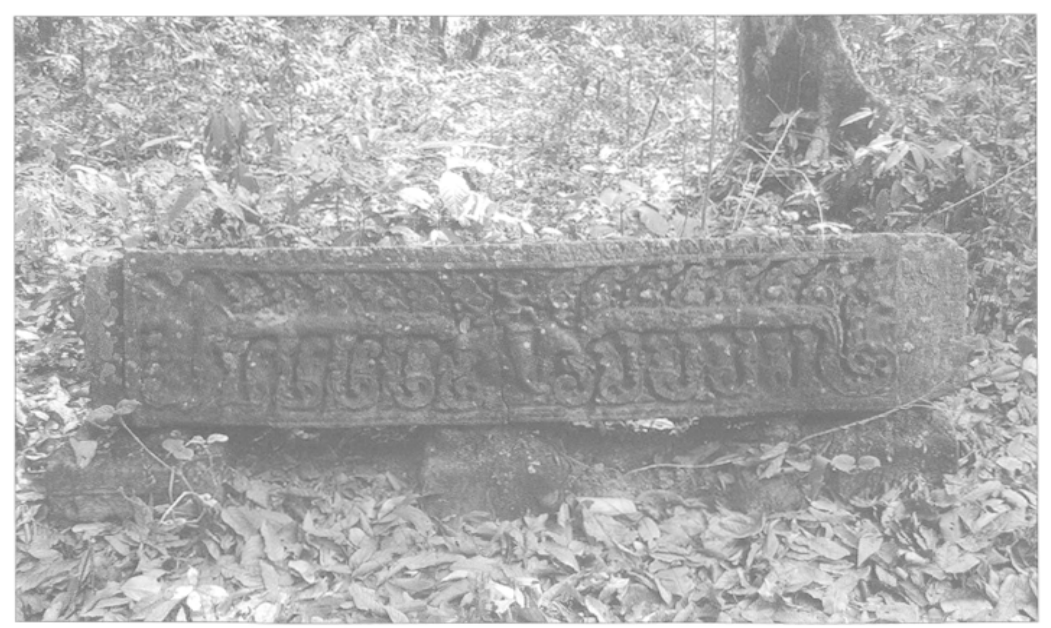

Fig. 6 : linteau décoratif découvert a Houay Tomo : grès ; $x^{\circ}-x^{\prime \prime}$ siècle de notre ère (cliché : Dominique Soutif). 


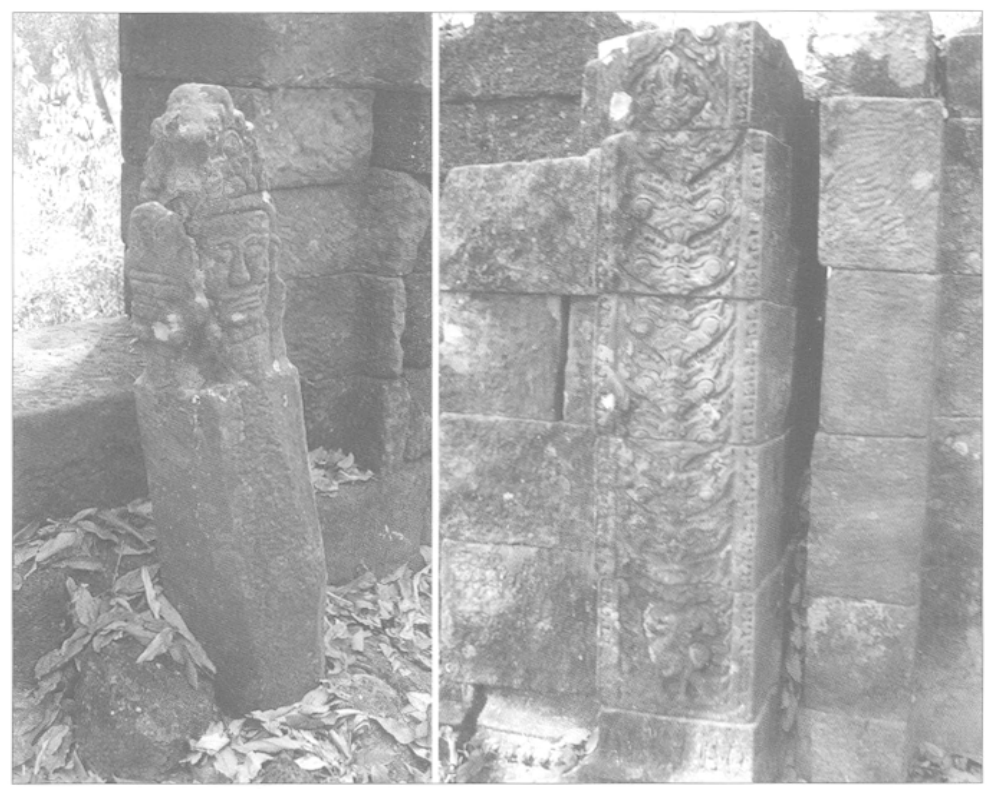

4 Fig. $7: 1$. borne śivaïte découverte dans le gopura Sud de Houay Tomo ; grès : période préangkorienne ?' (cliché : Dominique Soutif) : 2. pilastres du gopura Sud de Houay Tomo: grès ; fin du $\mathrm{XI}^{\mathrm{e}}$-début du $\times \|^{\mathrm{e}}$ siècle de notre ère (cliché : Dominique Soutif).
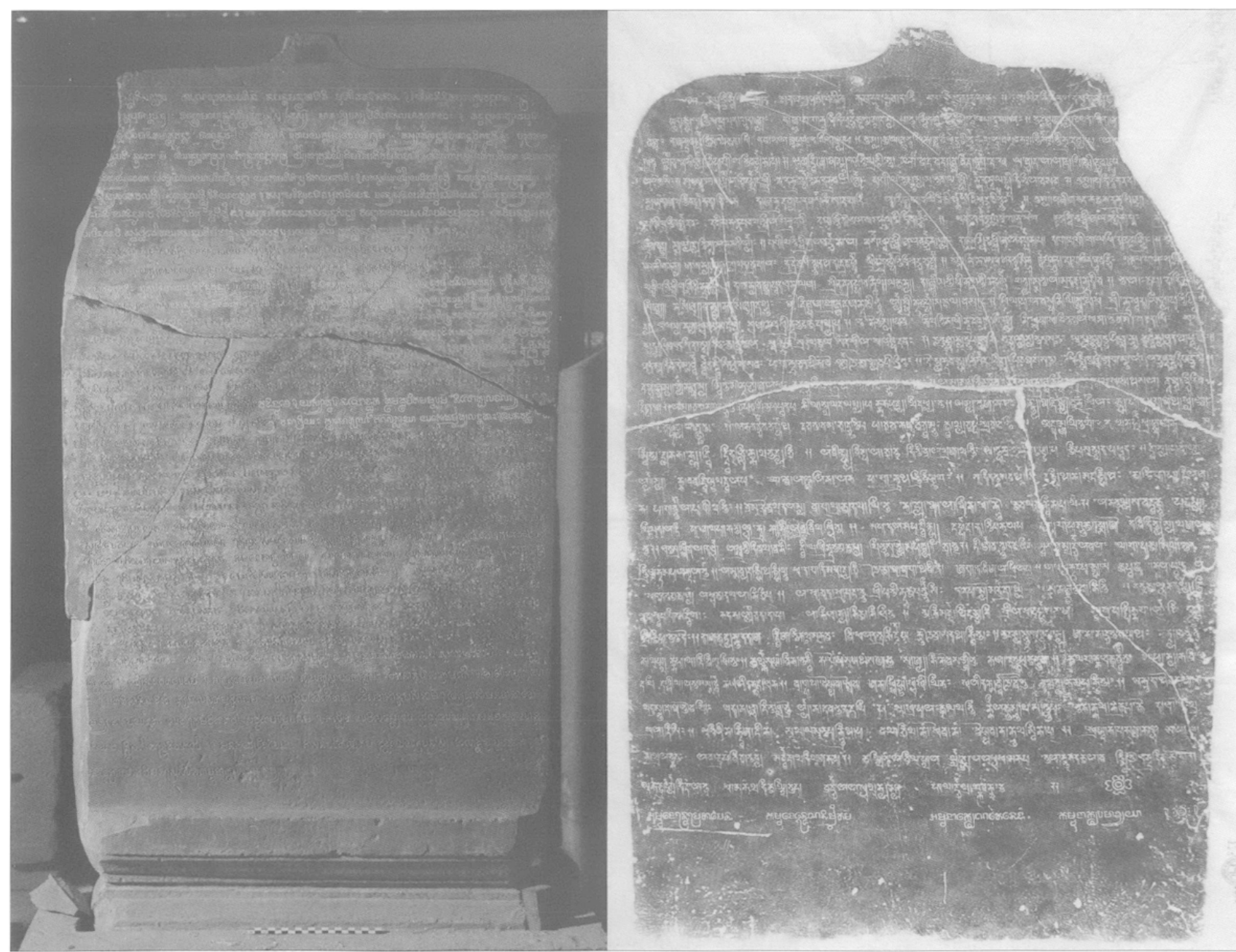

Fig. 8 : inscription K. 362 (face B) et son estampage (face A) : fin du $1 x^{\circ}$ siècle de notre ère : grès : $146.9 \times 74.5 \times 15.4 \mathrm{~cm}$ (clichés : Dominique Soutif \& EFFO AMPP 9817). 


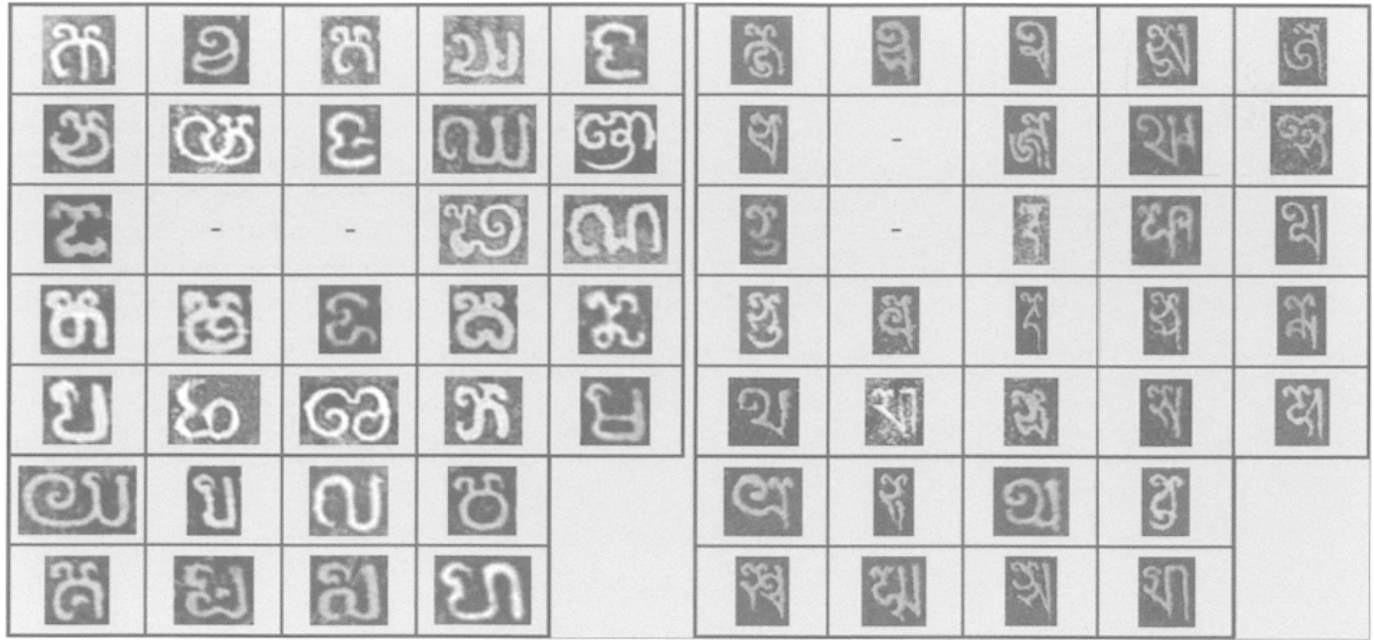

Fig. 9 : syllabaires des inscriptions digraphiques de Yaśovarman ${ }^{\mathrm{Fr}}$ (fin du $1 x^{\mathrm{e}}$ siècle de notre ère) : à gauche, les caractères khmers "classiques" : à droite. les caractères «étrangers" (estampages EFEO).

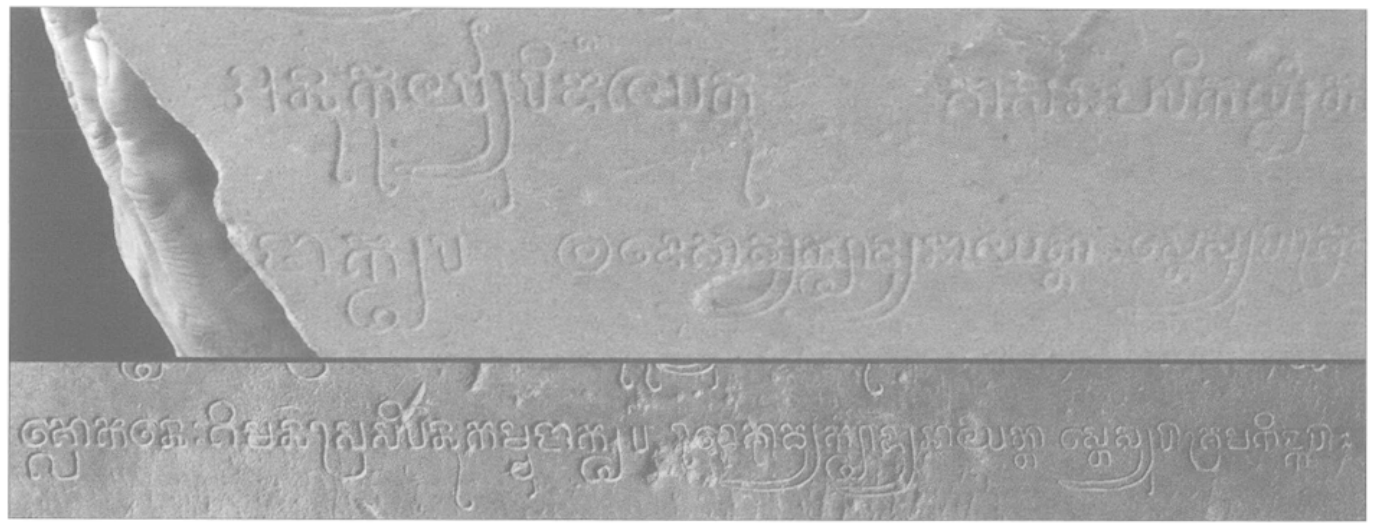

Fig. 10 : changement de gravure au niveau de la stance $L$ de la face B des inscriptions K. 1005 (en haut. cliché : Michel Lorrillard) et K. 362 (en bas, cliché : Dominique Soutif).

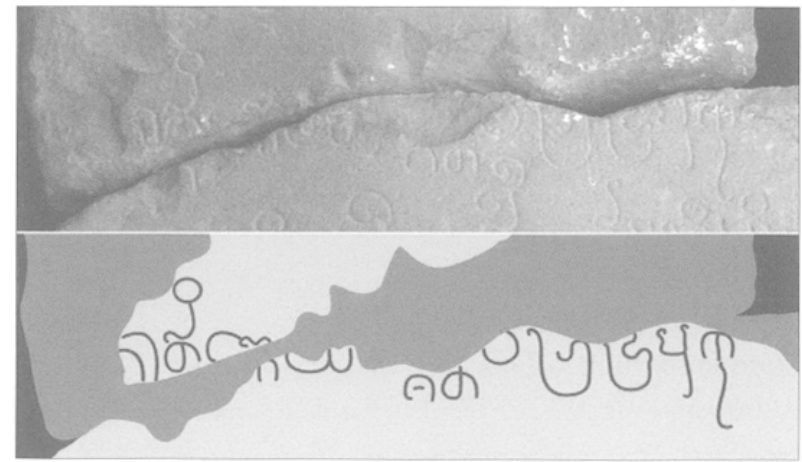

Fig. 11 : point de jonction des fragments VP I 149-2 et VP 1 452-3 au niveau de la stance XVI de la face B de K. 1005 (cliché : David Bazin ; vectorisation : Pierre Pichard \& Dominicue Soutif). 


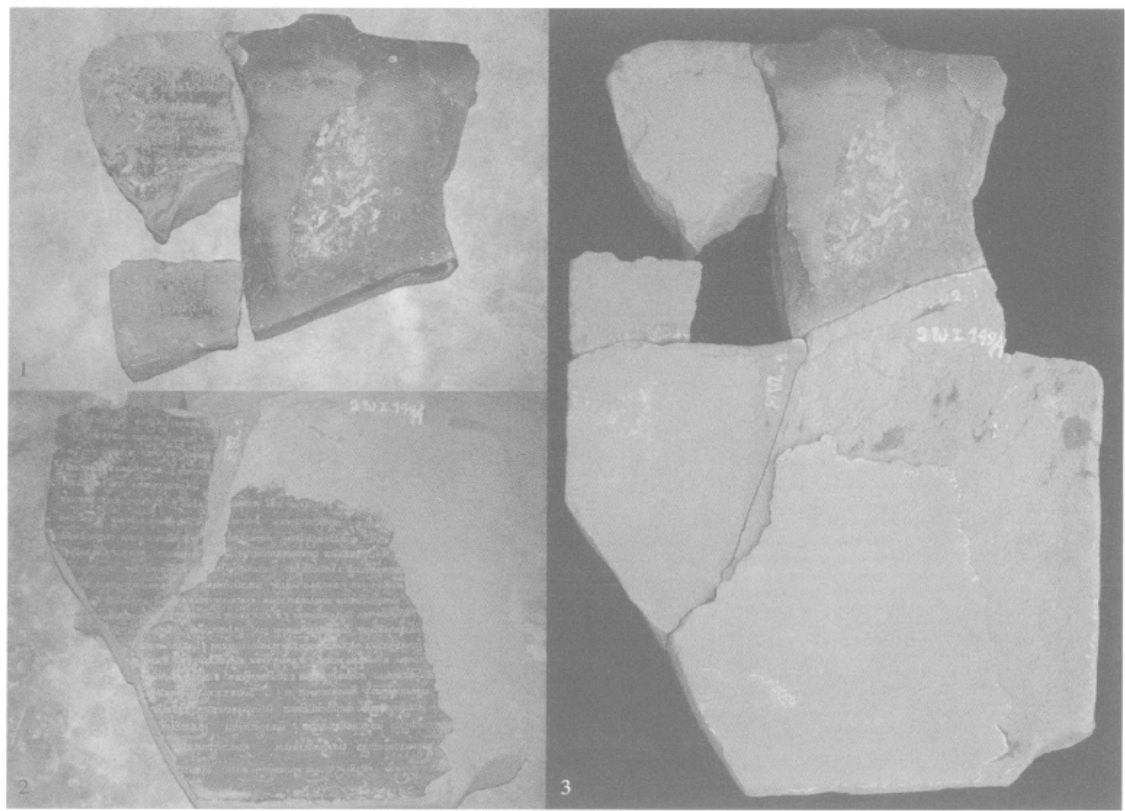

Fig. 12 : inscription K. 1005 : I. fragments VP I 452-1 à $3 ; 2$. VP I 149-1 à $2 ; 3$. K. 1005 reconstituée (clichés: Michel L.orrillard \& David Bazin). 